\title{
Enumeration of Vascular plants of Kalesar National Park, Yamuna Nagar, Haryana
}

\author{
A.N. Shukla', Durgesh Verma² and S.K. Srivastava ${ }^{3}$ \\ 'Botanical Survey of India, Central Regional Centre, Allahabad - 211002, Uttar Pradesh, India \\ ${ }^{2}$ Botanical Survey of India, Northern Regional Centre, Dehradun - 248195, Uttarakhand, India \\ ${ }^{3} B-1 / 146$, Sector 'G', Aliganj, Lucknow - 226024, Uttar Pradesh, India \\ Corresponding author: achutbsi@gmail.com
}

\section{कालेसर राष्ट्रीय उद्यान, यमुना नगर, हरियाणा के संवहनीय पादपजात की परिगणना} ए.एन.शुक्ला, दुर्गेश वर्मा एवं एस.के. श्रीवास्तव

\section{सारांश}

वर्तमान अध्ययन हरियाणा राज्य के यमुना नगर जनपद में स्थित कालेसर राष्ट्रीय उद्यान से अभिलेखित 256 वंशों एवं 80 कुलों की 317 जातियों का विवरण प्रस्तुत करता है। इनकें से 6 वंशों एवं 6 कुलों के अंतर्गत 6 जातियां पर्णागों से संबंधित हैं एवं पुष्पीय पौधों की 311 जातियां 250 वंशों में सवितरित हैं, जो 74 कुलों से संबंधित हैं। राष्ट्रीय उद्यान में पाये जाने वाले विभिन्न वानस्पतिक प्रकारों और विलुप्तप्राय जातियों का एक संक्षिप्त विवरण प्रस्तुत शोध पत्र में दिया गया है।

\section{Abstract}

The present study enumerates 317 taxa, distributed under 256 genera and 80 families that have neen recorded from the Kalesar National Park situated in Yamuna Nagar district of Haryana state. Of these, 6 species under 6 genera and 6 families belong to Pteridophytes and 311 flowering plant species distributed over 250 genera are belonging to 74 families. A brief account on the vegetation types and threatened species found in the National Park has been discussed.

Keywords: Flowering Plants, Haryana, Kalesar National Park, Pteridophytes, Threatened Species

\section{INTRODUCTION}

The state of Haryana has two National Parks namely Sultanpur National Park and Kalesar National Park and eight Wildlife Sanctuaries. No consolidated floristic account on the Kalesar National Park has been prepared hitherto. Botanical Survey of India, Northern Regional Centre, Dehradun has initiated inventerisation of the floral diversity in the Protected Areas of Haryana state under the Protected Area Network programme to bring out the comprehensive account of the floral diversity. Debta \& Srivastava (2015) documented the floral diversity of Sultanpur National Park. To document the diversity and occurrence of flowering plants in Kalesar National Park field explorations were conducted from 2013 to 2015 . The plants collected from various localities of the National Park in different seasons have been identified and all the processed and mounted specimens are housed in the herbarium of Botanical Survey of India, Dehradun (BSD) for reference. Kalesar National Park is located between $30^{\circ} 18^{\prime \prime}-30^{\circ} 27^{\prime \prime} \mathrm{N}$ and $77^{\circ} 25^{\prime \prime}-77^{\circ} 35^{\prime \prime} \mathrm{E}$ at the 
foothills of Shiwalik ranges of Yamuna Nnagar district, Haryana and sharing boundaries with three states namely Himachal Pradesh, Uttarakhand and Uttar Pradesh. The National Park was established on 8th December 2003. It was named after the Kaleshra (Shiva) temple located in the National Park. The total geographical area of the National Park is about $46 \mathrm{~km}^{2}$, and the elevation ranges from $609 \mathrm{~m}$ to $1066 \mathrm{~m}$. The National Park supports dense Sal forest (Shorea robusta), Khair forest (Senegalia catechu) and patches of grasslands, which support variety of plants and animals. Kalesar has 53\% dense forest, $38 \%$ open forest and $9 \%$ scrub. Total forest cover is about $71 \%$, and is the only Sal forest zone in the entire state of Haryana. Study of literature indicates that very little attention has been paid to study the flora of Kalesar National Park (Jain, 1985; Jain \& al., 2000; Kumar, 2001; Jain, 2004; Kumar \& Nagiyan, 2006).

\section{VEGETATION TYPES}

\section{Sal Forest}

The sal (Shorea robusta) forest occupies a major part of Kalesar. The common associates of Shorea robusta are Adina cordifolia, Ficus racemosa, Ficus semicordata, Holarrhena pubescens, Holoptelea integrifolia, Mallotus philippensis, Millettia extensa, Mitragyna parvifolia, Senegalia catechu, Terminalia elliptica, etc. The shrubby layer is composed of species such as Clerodendrum infortunatum, Justicia adhatoda, Helicteres isora, Carissa spinarum, Woodfordia fruticosa, Desmostachya bipinnata, Flemingia macrophylla, etc. Some of the climbers like Phanera vahlii are also found in the sal forest. The common herbs are Alysicarpus vaginalis, Crotalaria spectabilis, Evolvulus alsinoides, Apluda mutica, Eleusine coracana, Saccharum spontaneum, Ageratum conyzoides, etc.

\section{Scrub forest}

These forests occur on exposed steep hill slopes and ridges, where drier conditions prevail. The biotic interferences and excessive grazing pressure has resulted in sparse tree layers and discontinuous vegetation, while shrubs become comparatively dense. These are characterized by stunted growth and many stemmed trees and shrubs with small thorny bushes. The common species recorded in this area are Butea monosperma, Carissa spinarum, Euphorbia neriifolia, Flacourtia indica, Mimosa rubicaulis subsp. himalayana, Nyctanthes arbor-tristis, Premna barbata,
Premna mollissima, Senegalia catechu, Senegalia gageana, Senegalia torta, Woodfordia fruticosa, etc.

\section{Miscellaneous Forest}

The miscellaneous forest occupies large stretches of land of Kalesar National Park. Important constituents of this forest are: Senegalia catechu, Butea monosperma, Dalbergia sissoo, Ficus semicordata, Garuga pinnata, Helicteres isora, Mitragyna parvifolia, Nyctanthes arbortristis, Sterculia villosa, Terminalia elliptica, etc.

Threatened species: Rauvolfia serpentina (Critically endangered), Oroxylum indicum (Vulnerable).

\section{FLORISTIC ANALYSIS}

Intensive and extensive field surveys conducted in the National Park have resulted in the documentation of 317 plant species, distributed in 256 genera belonging to 80 families. Of these, 6 species in 6 genera and 6 families belong to Pteridophytes and 311 species of angiosperms distributed over 250 genera and belonging to 74 families. Among them, the dicotyledonous flora represents 263 species from 213 genera belonging to 62 families and the monocotyledonous flora with 48 species from 37 genera belonging to 12 families. The family Fabaceae has the maximum number of species (27 species), followed by Asteraceae (23 species), Poaceae (22 species), Malvaceae (16 species), Rubiaceae (12 species), Euphorbiaceae (11 species), Acanthaceae (11 species), Scrophulariaceae (10 species), Cyperaceae (10 species), and Solanaceae (9 species) (Fig. 1). Some of the dominant genera in the area are Cyperus (6 Species), Solanum and Grewia (5 species each), Eragrostis, Terminalia and Sida (4 species each), Ziziphus, Euphorbia, Crotalaria and Ficus (3 species

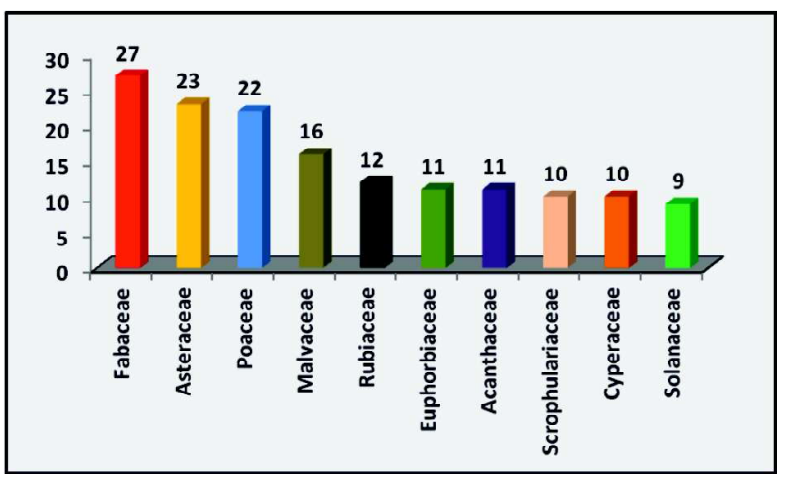

Fig. 1. Ten largest families in the flora of K.N.P. 
each) (Fig. 2). Among all taxa, 181 species (57\%) are herbs, 68 species $(21 \%)$ are trees, 43 species ( $14 \%)$ are shrubs, and 25 species ( $8 \%$ ) are climbers (Fig. 3 ).

The enumeration of plants provides the correct name of every taxon with complete protologue citation, basionym (if present) with citation, habitat and list of specimen(s) examined.

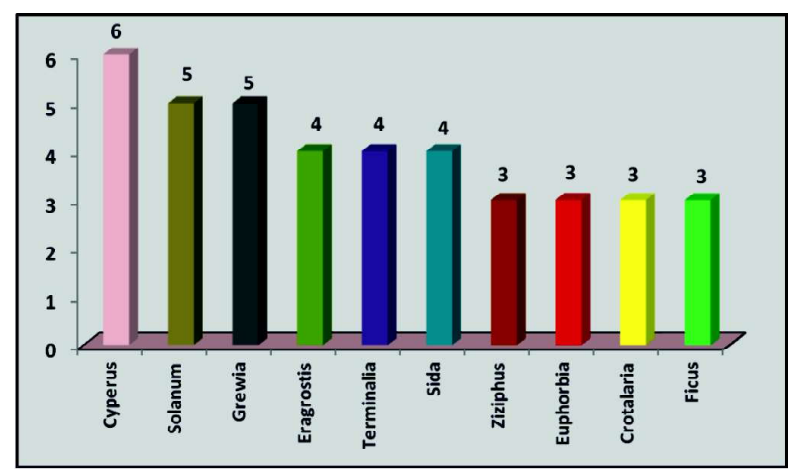

Fig. 2. Ten largest genera in the flora of KNP

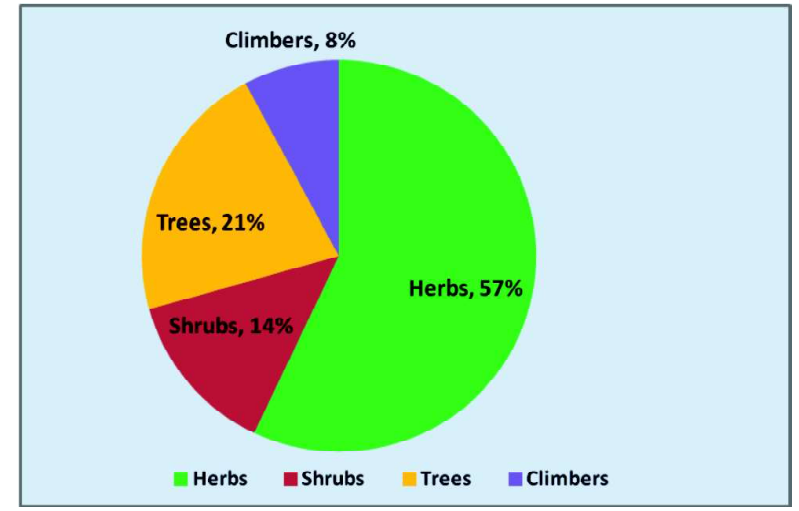

Fig. 3. Habit-wise distribution of taxa in KNP

\section{ENUMERATION OF PLANTS}

\section{PTERIDOPHYTE}

\section{ADIANTACEAE}

Adiantum capillus-veneris L., Sp. Pl.: 1096. 1753.

On moist places; Kalesar National Park, Compartment 18, 15.11.2014, A.N. Shukla \& S.K. Srivastava 122777.

\section{EQUISETACEAE}

Equisetum ramosissimum Desf., Fl. Atlant. 2: 398. 1799.
On moist places along nala; Kalesar National Park, Compartment 17, 04.03.2014, A.N. Shukla \& S.K. Srivastava 122109; Along Bara River, 13.08.2013, A.N. Shukla \& S.K. Srivastava 122053; Compartment 18, 15.11.2014, A.N. Shukla \& S.K. Srivastava 122781.

\section{LYGODIACEAE}

Lygodium flexuosum (L.) Sw., J. Bot. (Schrader) 1800(2): 106. 1801. Ophioglossum flexuosum L., Sp. Pl. 1063. 1753.

On roadsides; Kalesar National Park, near Forest Rest House, 20.11.2014, A.N. Shukla \& S.K. Srivastava 123947.

\section{MARSILEACEAE}

Marsilea minuta L., Mant. Pl.: 308. 1771.

In lakes; Kalesar National Park, along Yamuna River, 10.03.2014, A.N. Shukla \& S.K. Srivastava 122735.

\section{SINOPTERIDACEAE}

Cheilanthes bicolor Fraser-Jenk., Pakistan Syst. 5(12): 94.1992.

On moist slopes; Kalesar National Park., Compartment 18, 15.11.2014, A.N. Shukla \& S.K. Srivastava 122786.

\section{THELYPTERIDACEAE}

Ampelopteris prolifera (Retz.) Copel., Gen. Fil.: 144. 1947. Hemionitis prolifera Retz., Observ. Bot. 6: 3. 1791.

On moist places; Kalesar National Park, Compartment 18, 15.11.2014, A.N. Shukla \& S.K. Srivastava 122776.

\section{ANGIOSPERMS}

\section{RANUNCULACEAE}

Clematis gouriana Roxb. ex DC., Syst. Nat. 1: 138. 1817.

On dry places, climbing on tree.

Flowering and fruiting: September-October; Kalesar National Park, along roadside near Kalesar temple, 07.03.2014, A.N. Shukla \& S.K. Srivastava 122144; Near Kalesar village, 18.11.2014, A.N. Shukla \& S.K. Srivastava 123910.

Ranunculus sceleratus L., Sp. Pl.: 551. 1753.

On moist places. 
Flowering and fruiting: December-March; Kalesar National Park, Along Yamuna River, 09.03.2014, A.N. Shukla \& S.K. Srivastava 122706.

\section{ANNONACEAE}

Miliusa velutina (Dunal) Hook.f. \& Thomson, Fl. Ind.: 151. 1855 \& in Hook.f., Fl. Brit. India 1: 87. 1872. Uvaria velutina Dunal, Monogr. Anonac.: 91. 1817.

In sal and miscellaneous forests.

Flowering and fruiting: March-August; it is reported here on the basis of Negi \& al. (l.c.).

\section{MENISPERMACEAE}

Cissampelos pareira L. var. hirsuta (Buch.-Ham. ex DC.) Forman, Kew Bull. 22: 356. 1968. Cissampelos hirsuta Buch.-Ham. ex DC., Syst. Nat. 1: 535. 1817.

Along roadsides and in the forests.

Flowering and fruiting: July-October; Kalesar National Park, Compartment - 15, 07.08.2013, A.N. Shukla \& S.K. Srivastava 121918; Compartment - 22, 16.11.2014, A.N. Shukla \& S.K. Srivastava 122797.

\section{PAPAVERACEAE}

Argemone mexicana L., Sp. Pl.: 508. 1753.

On sandy places.

Flowering and fruiting: January-December; Kalesar National Park, along Yamuna River, 09.03.2014, A.N. Shukla \& S.K. Srivastava 122191.

\section{FUMARIACEAE}

Fumaria indica (Hausskn.) Pugsley, J. Linn. Soc., Bot. 44: 313. 1919. F. vaillantii Loisel. var. indica Hausskn. in Flora 56: 443. 1873.

On moist places.

Flowering and fruiting: January-March; Kalesar National Park, Compartment - 3, Fire line, 08.03.2014, A.N. Shukla \& S.K. Srivastava 122162; Along Yamuna River, 09.03.2014, A.N. Shukla \& S.K. Srivastava 122169.

\section{BRASSICACEAE}

Brassica juncea (L.) Czern., Conspect. Pl. Charc.: 8. 1859. Sinapis juncea L., Sp. Pl.: 668. 1753.

On roadsides.
Flowering and fruiting: December-March; Kalesar National Park, near Kalesar temple, 07.03.2014, A.N. Shukla \& S.K. Srivastava 122154.

Brassica rapa L., Sp. Pl.: 666. 1753.

On moist sandy places.

Flowering and fruiting: January-March; Kalesar National Park, Compartment - 1, 06.03.2014, A.N. Shukla \& S.K. Srivastava 122138.

Capsella bursa-pastoris (L.) Medik., Pfl.-Gatt.: 85. 1792. Thlaspi pastoris L., Sp. Pl.: 647. 1753.

On moist sandy places.

Flowering and fruiting: January-April; Kalesar National Park, Compartment - 1, 06.03.2014, A.N. Shukla \& S.K. Srivastava 122140.

Cardamine flexuosa With., Arr. Brit. Pl., ed. 3, 3: 578. 1796.

On moist places.

Flowering and fruiting: January-August; Kalesar National Park, near Kalesar village, 09.03.2014, A.N. Shukla \& S.K. Srivastava 122167.

Lepidium didymum L., Mant. Pl.: 92. 1767.

On moist places.

Flowering and fruiting: December-March; Kalesar National Park, along Yamuna River, 10.03.2014, A.N. Shukla \& S.K. Srivastava 122721, 122725.

Nasturtium officinale W.A. Aiton, Hort. Kew., ed. 2, 4: 110.1812.

On marshy places, lake margins.

Flowering and fruiting: February-March; Kalesar National Park, along Yamuna river, 10.03.2014, A.N. Shukla \& S.K. Srivastava 122713, 122716.

Raphanus raphanistrum L. subsp. sativus (L.) Domin, Beih. Bot. Centralbl. 26(2): 255. 1910. R. sativus L., Sp. Pl.: 669. 1753.

On roadsides.

Flowering and fruiting: December-April; Kalesar National Park, near forest rest house, 05.03.2014, A.N. Shukla \& S.K. Srivastava 122122. 


\section{FLACOURTIACEAE}

Casearia tomentosa Roxb., Fl. Ind., ed. 2: 421. 1824.

In dry deciduous forest.

Flowering and fruiting: April-August; it is reported here on the basis of Kumar (l.c.).

Flacourtia indica (Burm.f.) Merr., Interpr. Herb. Amboin.: 377. 1917. Gmelina indica Burm.f., Fl. Ind.: 132 , t. 39 , f. 5.1768 .

Common in dry deciduous forest.

Flowering and fruiting: March-August; it is reported here on the basis of Kumar (l.c.).

\section{POLYGALACEAE}

Polygala erioptera DC., Prodr. 1: 326. 1824.

On roadsides and moist slopes.

Flowering and fruiting: September-March; Kalesar National Park, Compartment - 17, 11.08.2013, A.N. Shukla \& S.K. Srivastava 122020.

\section{CARYOPHYLLACEAE}

Arenaria serpyllifolia L., Sp. Pl.: 423. 1753.

On fireline in moist places.

Flowering and fruiting: February-March; Kalesar National Park, Compartment - 2, 07.03.2014, A.N. Shukla \& S.K. Srivastava 122145.

Spergula arvensis L., Sp. Pl.: 440. 1753.

On moist places.

Flowering and fruiting: October-March; Kalesar National Park, along Yamuna River, 09.03.2014, A.N. Shukla \& S.K. Srivastava 122705.

Stellaria media (L.) Vill, Hist. Pl. Dauphiné 3: 615. 1789. Alsine media L., Sp. Pl.: 272. 1753.

On moist places.

Flowering and fruiting: November-March; Kalesar National Park, Compartment - 17, 04.03.2014, A.N. Shukla \& S.K. Srivastava 122112; Kalesar National Park, near Kalesar village, 09.03.2014, A.N. Shukla \& S.K. Srivastava 122165.

\section{PORTULACACEAE}

Portulaca pilosa L., Sp. Pl.: 445. 1753.

On moist places.
Flowering and fruiting: October-January; Kalesar National Park, along Yamuna River, 09.03.2014, A.N. Shukla \& S.K. Srivastava 122172.

\section{ROSACEAE}

Potentilla supina L., Sp. Pl.: 497. 1753.

On moist places.

Flowering and fruiting: November-March; Kalesar National Park, along Yamuna River, 09.03.2014, A.N. Shukla \& S.K. Srivastava 122173.

\section{DIPTEROCARPACEAE}

Shorea robusta C.F. Gaertn., Suppl. Carp.: 48, t. 186. 1805.

Common in plains.

Flowering and fruiting: February-July; it is reported here on the basis of Jain \& al. (l.c.).

\section{MALVACEAE}

Abutilon ramosum (Cav.) Guill. \& Perr., Fl. Seneg. Tent.: 68. 1831. Sida ramosa Cav., Diss. 1: 28, t. 6, f. 1. 1785.

In mixed forest.

Flowering and fruiting: October-December; Kalesar National Park, on border of N.P., 26.11.2014, A.N. Shukla \& S.K. Srivastava 123943.

Corchorus aestuans L., Syst. Nat., ed. 10, 2: 1079. 1759.

Common in open exposed places and on roadsides.

Flowering and fruiting: May-October; reported here on the basis of Negi \& al. (l.c.)

Helicteres isora L., Sp. Pl.: 963. 1753.

On dry slopes.

Flowering and fruiting: July-January; Kalesar National Park, Compartment - 21, 16.11.2014, A.N. Shukla \& S.K. Srivastava 122792.

Grewia flavescens Juss., Ann. Mus. Natl. Hist. Nat. 4: 91. 1804.

Rare in Kalesar reserve forest.

Flowering and fruiting: April-June; reported here on the basis of Jain \& al. (l.c.).

Grewia hirsuta Vahl, Symb. Bot. 1: 84. 1790.

In dry forest. 
Flowering and fruiting: August-October; Kalesar National Park, Compartment - 18, 15.11.2014, A.N. Shukla \& S.K. Srivastava 122784.

Grewia optiva J.R. Drumm. ex Burret, Notizbl. Bot. Gart. Berlin-Dahlem 9: 692. 1926.

Common in hilly tracts.

Flowering and fruiting: April-November; reported here on the basis of Jain \& al. (l.c.).

Grewia serrulata DC., Prodr. 1: 510. 1824.

Rare in reserve forest.

Flowering and fruiting: September-October; reported here on the basis of Jain \& al. (l.c.).

Grewia tiliifolia Vahl, Symb. Bot. 1: 35. 1790.

Rare in reserve forest.

Flowering and fruiting: August-December; reported here on the basis of Jain \& al. (l.c.).

Kydia calycina Roxb., Pl. Coromandel 3: 11, t. 215. 1819.

On stony slopes.

Flowering and fruiting: July-December; Kalesar National Park, Compartment - 24, 17.11.2014, A.N. Shukla \& S.K. Srivastava 123901.

Malvastrum coromandelianum (L.) Garcke, Bonplandia (Hannover) 5: 297. 1857. Malva coromandeliana L., Sp. Pl.: 687. 1753.

On roadsides.

Flowering and fruiting: August-October; Kalesar National Park, Compartment - 3, 08.03.2014, A.N. Shukla \& S.K. Srivastava 122159.

Sida acuta Burm.f., Fl. Ind.: 147. 1768.

On roadsides.

Flowering and fruiting: September-March; Kalesar National Park, Compartment - 15, 07.08.2013, A.N. Shukla \& S.K. Srivastava 121919; Near Kalesar village, 08.08.2013, A.N. Shukla \& S.K. Srivastava 121938; Near Kalesar temple, 19.11.2014, A.N. Shukla \& S.K. Srivastava 123930.

Sida cordata (Burm.f.) Borss.Waalk. Blumea 14: 182. 1966. Melochia cordata Burm.f., Fl. Ind.: 143. 1768.

On dry sandy places.
Flowering and fruiting: February-July; Kalesar National Park, Compartment - 15, along road side, 07.08.2013, A.N. Shukla \& S.K. Srivastava 121904; Compartment - 17, 12.03.2014, A.N. Shukla \& S.K. Srivastava 122742; Compartment - 18, 15.11.2014, A.N. Shukla \& S.K. Srivastava 122785.

Sida cordifolia L., Sp. Pl.: 684. 1753.

Along river bank.

Flowering and fruiting: September-October; Kalesar National Park, along Sokra village, 10.08.2013, A.N. Shukla \& S.K. Srivastava 121976.

Sida rhombifolia L., Sp. Pl.: 684. 1753.

Sandy places.

Flowering and fruiting: August-December. Kalesar National Park, Compartment - 14, 10.08.2013, A.N. Shukla \& S.K. Srivastava 121998.

Sterculia villosa Roxb. ex Sm. in Rees, Cycl. (London ed.) 34: Sterculia no. 16. 1816 .

On dry places.

Flowering and fruiting: April-July; Kalesar National Park, Compartment - 17, 12.03.2014, A.N. Shukla \& S.K. Srivastava 122747.

Urena lobata L., Sp. Pl.: 692. 1753.

On semi-moist places.

Flowering and fruiting: September-December; Kalesar National Park, Compartment - 13, 12.08.2013, A.N. Shukla \& S.K. Srivastava 122038.

\section{GERANIACEAE}

Geranium rotundifolium L., Sp. Pl.: 683. 1753.

In fire line grassland.

Flowering and fruiting: April-June; Kalesar National Park, Compartment - 2, 07.03.2014, A.N. Shukla \& S.K. Srivastava 122155.

\section{OXALIDACEAE}

Biophytum sensitivum (L.) DC., Prodr. 1: 690. 1824. In shady places.

Flowering and fruiting: August-November; reported here on the basis of Kumar (l.c.). 
Oxalis corniculata L., Sp. Pl.: 435. 1753.

On moist places.

Flowering and fruiting: Throughout the year; Kalesar National Park, Compartment - 21, 05.03.2014, A.N. Shukla \& S.K. Srivastava 122121.

\section{RUTACEAE}

Aegle marmelos (L.) Corrêa, Trans. Linn. Soc. London 5: 223. 1800. Crateva marmelos L., Sp. Pl.: 444. 1753.

In dry forest.

Flowering and fruiting: April-August; Kalesar National Park, Compartment - 15, 07.08.2013, A.N. Shukla \& S.K. Srivastava 121908.

Murraya koenigii (L.) Spreng., Syst. Veg. 2: 315. 1817. Bergera königii L., Mant. Pl.: 563. 1771.

Occasionally found in waste places.

Flowering and fruiting: April-June; reported here on the basis of Kumar (l.c.).

Murraya paniculata (L.) Jack, Malayan Misc. 1(5): 31. 1820. M. exotica L., Mant. Pl.: 68. 1767.

Rare on hill tracts.

Flowering and fruiting: April-September; reported here on the basis of Jain \& al. (l.c.).

\section{Burseraceae}

Boswellia serrata Roxb. ex Colebr., Asiat. Res. 9: 379, t. 5.1807.

On dry hills and slopes.

Flowering and fruiting: April-June; reported here on the basis of Jain \& al. (l.c.).

Garuga pinnata Roxb., Pl. Coromandel 3: 5, t. 208. 1811.

Rare in dry deciduous forests.

Flowering and fruiting: February-May; reported here on the basis of Kumar (l.c.).

\section{MELIACEAE}

Melia azedarach L., Sp. Pl.: 384. 1753.

On dry places.

Flowering and fruiting: March-September. Kalesar National Park, along Yamuna River, 09.03.2014, A.N. Shukla \& S.K. Srivastava 122187.

\section{RHAMNACEAE}

Helinus lanceolatus Brandis, Forest Fl. N.W. India: 574. 1874.

Occasional on ravine slopes.

Flowering and fruiting: October-April; reported here on the basis of Kumar (l.c.).

Ventilago denticulata Willd., Neue Schriften Ges. Naturf. Freunde Berlin 3: 417.

1801.

In moist riverian localities.

Flowering and fruiting: June-July; reported on the basis of Negi (l.c.).

Ziziphus jujuba Mill., Gard. Dict., ed. 81. 1768.

On dry slopes.

Flowering and fruiting: September-October. Kalesar National Park, Compartment - 18, 15.11.2014, A.N. Shukla \& S.K. Srivastava 122772.

Ziziphus oenopolia (L.) Mill., Gard. Dict., ed. 8, n. 3. 1768. Rhamnus oenopolia L., Sp. Pl.: 194. 1753.

On roadsides, dry slopes, climbing on tree.

Flowering and fruiting: December-May; Kalesar National Park, along nala in compartment - 17, 11.08.2013, A.N. Shukla \& S.K. Srivastava 122016; Along Yamuna River, 09.03.2014, A.N. Shukla \& S.K. Srivastava 122188; Near Kalesar temple, 10.03.2014, A.N. Shukla \& S.K. Srivastava 122733; Compartment - 18, 15.11.2014, A.N. Shukla \& S.K. Srivastava 122779.

Ziziphus xylopyrus (Retz.) Willd., Sp. Pl. 1: 1104. 1798. Rhamnus xylopyrus Retz., Observ. Bot. 2: 11. 1781.

On roadsides.

Flowering and fruiting: April-January. Kalesar National Park, Compartment - 17, 11.08.2013, A.N. Shukla \& S.K. Srivastava 122015.

\section{SAPINDACEAE}

Schleichera oleosa (Lour.) Oken, Allg. Naturgesch. 3(2): 1341. 1841. Pilosa oleosa Lour., Fl. Cochinch.: 615. 1790 .

On dry slopes.

Flowering and fruiting: July-August; reported here on 
the basis of Negi (l.c.).

\section{ANACARDIACEAE}

Buchanania cochinchinensis (Lour.) M.R. Almeida, Fl. Maharashtra 1:287. 1996. Toluifera cochinchinensis Lour., Fl. Cochinch. 1: 262. 1790.

In mixed forest.

Flowering and fruiting: January-June; Kalesar National Park, border of N.P., 20.11.2014, A.N. Shukla \& S.K. Srivastava 123944.

Lannea coromandelica (Houtt.) Merr., J. Arnold Arbor. 19(4): 353. 1938. Dialium coromandelicum Houtt., Nat. Hist. 2: 39, t. 5, f. 2. 1774.

In miscellaneous forest.

Flowering and fruiting: June-August; reported here on the basis of Negi (l.c.).

Spondias pinnata (L.f.) Kurz, Prelim. Rep. Forest Pegu App. A: 44. 1875. Mangifera pinnata L.f., Suppl. Pl.: 156. 1781.

Occasional in forests.

Flowering and fruiting: April-June; reported here on the basis of Kumar (l.c.).

\section{FABACEAE}

Abrus precatorius L., Syst. Nat., ed. 12, 2: 472.1767.

On roadsides.

Flowering and fruiting: September-October; Kalesar National Park, near Kalesar village, 08.08.2013, A.N. Shukla \& S.K. Srivastava 121945.

Alysicarpus ovalifolius (Schumach.) J. Léonard, Bull. Jard. Bot. État Bruxelles 24: 88. 1954. Hedysarum ovalifolium Schumach., Beskr. Guin. Pl.: 359. 1827.

On riverside.

Flowering and fruiting: September-January; Kalesar National Park, near Kalesar village, 08.08.2013, A.N. Shukla \& S.K. Srivastava 121944.

Alysicarpus vaginalis (L.) DC., Prodr. 2: 353. 1825. Hedysarum vaginale L., Sp. Pl.: 746. 1753.

On rocky slopes.

Flowering and fruiting: September-October; Kalesar National Park, Compartment - 24, 17.11.2014, A.N. Shukla \& S.K. Srivastava 123909.
Butea monosperma (Lam.) Taub., Engl. \& Prantl, Nat. Pflanzenfam. 3(3): 366. 1894. Erythrina monosperma Lam., Encycl. 1(2): 391. 1785.

In mixed forest.

Flowering and fruiting: March-April; Kalesar National Park, Compartment - 21, 16.11.2014, A.N. Shukla \& S.K. Srivastava 122791; Compartment - 24, 17.11.2014, A.N. Shukla \& S.K. Srivastava 123907.

Cajanus scarabaeoides (L.) Thouars, G.-F.Cuvier, Dict. Sci. Nat. 6: 167. 1806. Dolichos scarabaeoides L., Sp. Pl.: 726. 1753 .

As forest undergrowth.

Flowering and fruiting: September-December; Kalesar National Park, Compartment - 8, 14.11.2014, A.N. Shukla \& S.K. Srivastava 122769.

Canavalia gladiata (Jacq.) DC., Prodr. 2: 404.1825. Dolichos gladiatus Jacq., Icon. Pl. Rar. 3(1): 560. 1788.

On roadside bushes.

Flowering and fruiting: August-November; recorded on the basis of Kumar (l.c.).

Crotalaria albida B. Heyne ex Roth, Nov. Pl. Sp.: 333. 1821.

On dry sandy slopes.

Flowering and fruiting: September-November; Kalesar National Park, Compartment - 21, 05.03.2014, A.N. Shukla \& S.K. Srivastava 122115; Compartment - 18, 15.11.2014, A.N. Shukla \& S.K. Srivastava 122782.

Crotalaria prostrata Rottler ex Willd., Enum. Pl.: 744. 1809.

On dry slopes.

Flowering and fruiting: October-December; Kalesar N.P., along nala, Compartment - 15, 11.08.2013, A.N. Shukla \& S.K. Srivastava 122019.

Crotalaria spectabilis Roth, Nov. Pl. Sp.: 341. 1821.

Along river bank.

Flowering and fruiting: September-December; Kalesar National Park, Compartment - 7, 13.03.2014, A.N. Shukla \& S.K. Srivastava 122750; Compartment - 8, 14.11.2014, A.N. Shukla \& S.K. Srivastava 122756, 122768 .

Dalbergia sissoo Roxb. ex DC., Prodr. 2: 416. 1825.

In mixed forest. 
Flowering and fruiting: April-October; Kalesar National Park, near Forest Rest House, 14.11.2014, A.N. Shukla \& S.K. Srivastava 123939.

Erythrina suberosa Roxb., Fl. Ind. 3: 253. 1832.

In mixed forest.

Flowering and fruiting: March-May; reported here on the basis of Negi \& al. (l.c.).

Flemingia macrophylla (Willd.) Kuntze ex Merr., Philipp. J. Sci. Bot. 5(2): 130. 1910. Crotalaria macrophylla Willd., Sp. Pl. 3: 982. 1802.

Common on grassy slopes.

Flowering and fruiting: June-September; reported here on the basis of Negi \& al. (l.c.).

Flemingia strobilifera (L.) W.T. Aiton, Hort. Kew., ed. 2, 4: 350. 1812. Hedysarum strobiliferum L., Sp. Pl.: 764. 1753.

In fire line zone.

Flowering and fruiting: September-November; Kalesar National Park, Compartment - 2, 07.03.2014, A.N. Shukla \& S.K. Srivastava 122157.

Grona trifolia (L.) H. Ohashi \& K. Ohashi, J. Jap. Bot. 93: 117. 2018. Hedysarum triflorum L., Sp. Pl.: 749. 1753.

On roadsides.

Flowering and fruiting: April-October; Kalesar National Park, near Kalesar village, 08.08.2013, A.N. Shukla \& S.K. Srivastava 121943.

Indigofera cassioides Rottler ex DC., Prodr. 2: 225. 1825.

In forest undergrowth.

Flowering and fruiting: October-May; Kalesar National Park, Compartment - 7, 13.03.2014, A.N. Shukla \& S.K. Srivastava 122751.

Indigofera hochstetteri Baker, Oliv. \& al., Fl. Trop. Afr. 2: 101. 1871.

In open ground and also in cultivated field.

Flowering and fruiting: August-October; reported on the basis of Negi \& al. (l.c.).

Lathyrus aphaca L., Sp. Pl.: 729. 1753.

On sandy slopes.

Flowering and fruiting: December-March; Kalesar
National Park, along Yamuna River, 10.03.2014, A.N. Shukla \& S.K. Srivastava 122717.

Medicago polymorpha L., Sp. Pl.: 779. 1753.

On sandy places.

Flowering and fruiting: December-March; Kalesar National Park, along Yamuna River, 10.03.2014, A.N. Shukla \& S.K. Srivastava 122722.

Millettia extensa (Benth.) Benth. ex Baker in Hook.f., Fl. Brit. India 2: 109. 1876. Otosema extensa Benth. in Miq., Pl. Jungh.: 249. 1852.

In mixed forests.

Flowering and fruiting: March-December; Kalesar National Park, Compartment - 13, 12.08.2013, A.N. Shukla \& S.K. Srivastava 122029.

Ougeinia oojeinensis (Roxb.) Hochr. Bull. Soc. Bot. Genève 13 \& 14: 51. 1909. Dalbergia oojeinensis Roxb., Fl. Ind. 3: 220. 1832.

Not common in mixed forest.

Flowering and fruiting: March-May; reported here on the basis of Jain \& al. (l.c.).

Phyllodium pulchellum (L.) Desv., Mém. Soc. Linn. Paris 4: 324. 1826. Hedysarum pulchellum L., Sp. Pl.: 747. 1753.

As forest undergrowth in sandy places.

Flowering and fruiting: December-April; Kalesar National Park, Compartment - 13, 12.08.2013, A.N. Shukla \& S.K. Srivastava 122025; Compartment - 8, 14.11.2014, A.N. Shukla \& S.K. Srivastava 122757.

Polhillides velutina (Willd.) H. Ohashi \& K. Ohashi, J. Jap. Bot. 94: 72. 2019. Hedysarum velutinum Willd., Sp. Pl., ed. 4, 3(2): 1174. 1803.

Common in hill side forests.

Flowering and fruiting: August-October; reported here on the basis of Jain \& al. (l.c.).

Pueraria tuberosa (Roxb. ex Willd.) DC., Ann. Sci. Nat. (Paris) 4: 97. 1825. Hedysarum tuberosum Roxb. ex Willd., Sp. Pl. 3: 1197. 1803.

Not common, in mixed forest.

Flowering and fruiting: March-July; reported here on the basis of Jain \& al. (l.c.).

Tadehagi triquetrum (L.) H. Ohashi, Ginkgoana 1: 290. 1973. Hedysarum triquetrum L., Sp. Pl.: 746. 1753. 
Common in grassy places and sal forest.

Flowering and fruiting: April-September; reported here on the basis of Jain \& al. (l.c.).

Trifolium dubium Sibth., Fl. Oxon.: 231. 1794.

On sandy places.

Flowering and fruiting: October-January; Kalesar National Park, along Yamuna River, 10.03.2014, A.N. Shukla \& S.K. Srivastava 122723.

Uraria lagopodioides (L.) DC., Prodr. 2: 324. 1825. Hedysarum lagopodioides L., Sp. Pl.: 1198. 1753.

In the undergrowth of dry forest.

Flowering and fruiting: October-December; Kalesar National Park, Compartment - 8, 14.11.2014, A.N. Shukla \& S.K. Srivastava 122762.

Vicia sativa L., Sp. Pl.: 736. 1753.

On sandy slopes.

Flowering and fruiting: December-March; Kalesar National Park, along Yamuna River, 10.03.2014, A.N. Shukla \& S.K. Srivastava 122718.

\section{CAESALPINIACEAE}

Bauhinia racemosa Lam., Encycl. 1(2): 390. 1785.

In mixed forests.

Flowering and fruiting: June-November; reported here on the basis of Kumar (l.c.).

Bauhinia variegata L., Sp. Pl.: 375. 1753.

In sal mixed and mixed forests.

Flowering and fruiting: May-August; reported here on the basis of Negi \& al. (l.c.).

Chamaecrista absus (L.) H.S. Irwin \& Barneby Mem. New York Bot. Gard. 35: 664. 1982. Cassia absus L., Sp. Pl.: 376. 1753.

Common on hilly tracts.

Flowering and fruiting: August-October; reported here on the basis of Jain \& al. (l.c.).

Guilandina bonduc L., Sp. Pl.: 381. 1753.

On hilly tracts.

Flowering and fruiting: July-November; reported here on the basis of Jain \& al. (l.c.).

Phanera retusa Benth. in Miq., Pl. Jungh.: 263. 1852.
Common in the mixed forests on the hilly tracts.

Flowering and fruiting: September-December; reported here on the basis of Jain \& al. (l.c.).

Phanera vahlii (Wight \& Arn) Benth., Miq., Pl. Jungh.: 263. 1852. Bauhinia vahlii Wight \& Arn., Prodr. Fl. Ind. Orient. 1: 297. 1834.

Common in the sal and mixed forests.

Flowering and fruiting: April-October; reported here on the basis of Jain \& al. (l.c.).

Piliostigma malabaricum (Roxb.) Benth., Miq., Pl. Jungh.: 261. 1852. Bauhinia malabarica Roxb., Fl. Ind. 2: 321.1832

In mixed forest on rocky slopes and along river bank.

Flowering and fruiting: September-April; Kalesar National Park, Compartment - 17, 11.08.2013, A.N. Shukla \& S.K. Srivastava 122017; Near Forest Rest House, 13.08.2013, A.N. Shukla \& S.K. Srivastava 122042; Compartment - 17, 16.11.2014, A.N. Shukla \& S.K. Srivastava 122790; Compartment - 24, 17.11.2014, A.N. Shukla \& S.K. Srivastava 123906.

Senna occidentalis (L.) Link, Handbuch 2: 140. 1831. Cassia occidentalis L., Sp. Pl.: 377. 1753.

On roadsides.

Flowering and fruiting: March-November; Kalesar National Park, near Kalesar village, 18.11.2014, A.N. Shukla \& S.K. Srivastava 123921.

Tamarindus indica L., Sp. Pl.: 34. 1753.

Along road sides.

Flowering and fruiting: April-September; reported here on the basis of Jain \& al. (l.c.).

\section{MIMOSACEAE}

Mimosa rubicaulis Lam. subsp. himalayana (Gamble) H. Ohashi, Enum. Fl. Pl. Nepal 2: 126. 1979. Mimosa himalayana Gamble, Bull. Misc. Inform. Kew 1920: 4. 1920.

In mixed forest or on hillslopes.

Flowering and fruiting: July-December; reported here on the basis of Kumar (l.c.).

Senegalia catechu (L.f.) P.J.H. Hurter \& Mabb., Mabberley's Pl.-Book: 1021. 2008. Mimosa catechu L.f., Suppl. Pl.: 439. 1782.

On dry places. 
Flowering and fruiting: June-November; Kalesar National Park, Compartment - 17, 12.03.2014, A.N. Shukla \& S.K. Srivastava 122740.

Senegalia gageana (Craib) Maslin, Seigler \& Ebinger, Blumea 58: 40. 2013. Acacia gageana Craib, Bull. Misc. Inform. Kew 1915(10): 409. 1915.

Not common; on roadsides and exposed slopes.

Flowering and fruiting: March-August; reported here on the basis of Negi \& al. (l.c.).

Senegalia torta (Roxb.) Maslin, Seigler \& Ebinger, Blumea 58: 42. 2013. Mimosa torta Roxb., Fl. Ind. 2: 556. 1832 .

Rare in miscellaneous and mixed forests.

Flowering and fruiting: September-November; reported here on the basis of Jain \& al. (l.c.).

Pithecellobium dulce (Roxb.) Benth., London J. Bot. 3: 199. 1844. Mimosa dulcis Roxb., Pl. Coromandel 1(4): 67, t. 99. 1798.

Planted.

Flowering and fruiting: March-July; reported here on the basis of Kumar (l.c.).

Vachellia nilotica (L.) P.J.H. Hurter \& Mabb., Mabberley's Pl.-Book: 1021. 2008. Mimosa nilotica L., Sp. Pl.: 521. 1753.

On dry land.

Flowering and fruiting: January-November; Kalesar National Park, along Yamuna River, 10.03.2014, A.N. Shukla \& S.K. Srivastava 122734.

\section{COMBRETACEAE}

Terminalia anogeissiana Gere \& Boatwr., Bot. J. Linn. Soc. 184: 319. 2017.

In mixed forest.

Flowering and fruiting: August-March; Kalesar National Park, Compartment - 18, 14.11.2014, A.N. Shukla \& S.K. Srivastava 122766.

Terminalia arjuna (Roxb. ex DC.) Wight \& Arn., Prodr. Fl. Ind. Orient.: 34. 1834. Pentaptera arjuna Roxb. ex DC., Prodr. 3: 14. 1828.

Common on roadsides.

Flowering and fruiting: April-August; reported here on the basis of Jain \& al. (l.c.).
Terminalia bellirica (Gaertn.) Roxb., Pl. Coromandel 2(4): 54, t. 198. 1805. Myrobalnus bellirica Gaertn., Fruct. Sem. Pl. 2: 90, t. 97, f. a \& b. 1791.

Not common, along roadsides.

Flowering and fruiting: April-December; reported here on the basis of Jain \& al. (l.c.).

Terminalia elliptica Willd., Sp. Pl., ed. 4, 4: 969. 1806.

Common in dry deciduous forests.

Fl. \& F.: June-August; Kalesar National Park, Compartment - 21,05.03.2014, A.N. Shukla \& S.K. Srivastava 122118; Compartment - 7, 13.03.2014, A.N. Shukla \& S.K. Srivastava 122749.

\section{LYTHRACEAE}

Rotala rotundifolia (Buch.-Ham. ex Roxb.) Koehne, Bot. Jahrb. Syst. 1(2): 175. 1880. Ammannia rotundifolia Buch.-Ham. ex Roxb., Fl. Ind. 1: 446. 1820.

On roadsides.

Flowering and fruiting: November-April; Kalesar National Park, Compartment - 15, 10.08.2013, A.N. Shukla \& S.K. Srivastava 122000.

Woodfordia fruticosa (L.) Kurz, J. Asiat. Soc. Bengal, Pt. 2, Nat. Hist. 40: 56. 1871. Lythrum fruticosum L., Syst. Nat., ed. 10, 2: 1045. 1759.

On dry slopes.

Flowering and fruiting: January-May; Kalesar National Park, along Yamuna River, 09.03.2014, A.N. Shukla \& S.K. Srivastava 122708.

\section{ONAGRACEAE}

Ludwigia adscendens (L.) H. Hara, J. Jap. Bot. 28(10): 291. 1953. Jussiaea adscendens L., Mant. Pl.: 69. 1767.

On marshy places.

Flowering and fruiting: September-December; Kalesar National Park, along Yamuna River, 19.11.2014, A.N. Shukla \& S.K. Srivastava 123929.

Ludwigia hyssopifolia (G. Don) Exell, Garcia de Orta 5: 471. 1957. Jussiaea hyssopifolia G. Don, Gen. Hist. 2: 693.1832 .

On moist places.

Flowering and fruiting: October-May; Kalesar National Park, Compartment - 18, 15.11.2014, A.N. Shukla \& S.K. Srivastava 12773. 
Ludwigia octovalvis (Jacq.) P.H. Raven, Kew Bull. 15: 476, f. 6d-e, 1962. Oenothera octovalvis Jacq., Enum. Syst. Pl.: 19. 1760.

Occasionally found along water streams.

Flowering and fruiting: December-March; reported here on the basis of Jain \& al. (l.c.).

\section{PASSIFLORACEAE}

Passiflora foetida L., Sp. Pl.: 959. 1753.

On outskirts of forests.

Flowering and fruiting: August-November; Kalesar National Park, along Sokra River, 10.08.2013, A.N. Shukla \& S.K. Srivastava 121986.

\section{CUCURBITACEAE}

Citrullus colocynthis (L.) Schrad., Linnaea 12: 414. 1838. Cucumis colocynthis L., Sp. Pl.: 1011. 1753.

In sandy river-beds.

Flowering and fruiting: March-June; Kalesar National Park, along Yamuna River, 18.11.2014, A.N. Shukla \& S.K. Srivastava 123911; Near Kalesar village, 18.11.2014, A.N. Shukla \& S.K. Srivastava 123912.

Cucumis maderaspatana L., Sp. Pl.: 1012. 1753.

On sandy slopes.

Flowering and fruiting: September-February; Kalesar National Park, Compartment - 17, 11.08.2013, A.N. Shukla \& S.K. Srivastava 122023.

Solena amplexicaulis (Lam.) Gandhi in C.J. Saldanha \& Nicolson, Fl. Hassan Distr.: 179. 1976. Bryonia amplexicaulis Lam., Encycl. 1: 496. 1783.

On roadsides.

Flowering and fruiting: August-March; Kalesar National Park, Kanni line, 12.08.2013, A.N. Shukla \& S.K. Srivastava 122037.

Solena heterophylla Lour., Fl. Cochinch.: 514. 1790.

Common on hillocks.

Flowering and fruiting: August-March; reported here on the basis of Jain \& al. (l.c.).

Trichosanthes cucumerina L., Sp. Pl.: 1008. 1753.

Near habitations and in mixed forests.

Flowering and fruiting: September-January; Kalesar
National Park, near Kalesar village, 08.08.2013, A.N. Shukla \& S.K. Srivastava 121939.

\section{CACTACEAE}

Opuntia elatior Mill., Gard. Dict., ed. 8. n. 4. 1768.

Occasional in the forest and waste places.

Flowering and fruiting: October-December; reported here on the basis of Jain \& al. (l.c.).

\section{MOLLUGINACEAE}

Hypertelis cerviana (L.) Thulin, Taxon 65: 787. 2016. Pharnaceum cerviana L., Sp. Pl.: 272. 1753.

On moist places.

Flowering and fruiting: September; Kalesar National Park, along Yamuna River, 09.03.2014, A.N. Shukla \& S.K. Srivastava 122178.

\section{APIACEAE}

Bupleurum hamiltonii N.P. Balakr., J. Bombay Nat. Hist. Soc. 63: 328. 1967.

Along nala on slopes.

Flowering and fruiting: September-October; Kalesar National Park, Compartment - 17, 04.03.2014, A.N. Shukla \& S.K. Srivastava 122106.

\section{RUBIACEAE}

Adina cordifolia (Roxb.) Brandis, Forest Fl. N.W. India: 263. 1874. Nauclea cordifolia Roxb., Pl. Coromandel 1(3): 49, t. 53. 1796.

On roadsides.

Flowering and fruiting: May-June; Kalesar National Park, Kanni line, 372 m, 12.08.2013, A.N. Shukla \& S.K. Srivastava 122030.

Catunaregam spinosa (Thunb.) Triveng., Bull. Mus. Natl. Hist. Nat., Sér. 3, Bot. 35: 13. 1978. Gardenia spinosa Thunb., Diss. Gard. 7: 16, t. 2, f. 4. 1780.

Common on hilly tracts.

Flowering and fruiting: July-December; it is reported here on the basis of Jain \& al. (l.c.).

Galium aparine L., Sp. Pl.: 108. 1753.

On roadsides.

Flowering and fruiting: January-April; Kalesar National 
Park, near Kalesar village, 09.03.2014, A.N. Shukla \& S.K. Srivastava 122166.

Hymenodictyon orixense (Roxb.) Mabb., Taxon 31: 66. 1982. Cinchona orixensis Roxb., Bot. Descr. Swietenia 21. 1793.

On roadsides.

Flowering and fruiting: June-February; Kalesar National Park, one km from Forest Rest House, 363 m, 13.08.2013, A.N. Shukla \& S.K. Srivastava 122040; Compartment - 17, 16.11.2014, A.N. Shukla \& S.K. Srivastava 122789.

Knoxia sumatrensis (Retz.) DC., Prodr. 4: 569. 1830. Spermacoce sumantrensis Retz., Observ. Bot. 4: 23. 1786.

On moist places.

Flowering and fruiting: August-October; Kalesar National Park, Compartment - 8, 14.11.2014, A.N. Shukla \& S.K. Srivastava 122770.

Kohautia aspera (B. Heyne ex Roth) Bremek., Verh. Kon. Ned. Akad. Wetensch., Afd. Natuurk., Sect. 2, 48(2): 113. 1952. Hedyotis aspera B. Heyne ex Roth, Nov. Pl. Sp.: 94. 1821.

Common in sandy places and grassy localities.

Flowering and fruiting: August-November; it is reported here on the basis of Kumar (l.c.).

Mitragyna parvifolia (Roxb.) Korth., Observ. Naucl. Indic. 19 1839. Nauclea parvifolia Roxb., Pl. Coromandel 1(2): 40, t. 52. 1795.

On sandy slopes.

Flowering and fruiting: May-December; Kalesar National Park, near Forest Rest House, 09.03.2014, A.N. Shukla \& S.K. Srivastava 122194.

Morinda coreia Buch.-Ham., Trans. Linn. Soc. London 13: 537. 1822.

In mixed forest.

Flowering and fruiting: August-December; reported here on the basis of Jain \& al. (l.c.).

Scleromitrion verticillatum (L.) R.J. Wang, J. Trop. Subtrop. Bot. 22(5): 440.2014. Oldenlandia verticillata L., Mant. Pl. 1: 40.1767.

On moist places.

Flowering and fruiting: May-September; Kalesar
National Park, along Sokra River, 10.08.2013, A.N. Shukla \& S.K. Srivastava 121981; Compartment - 8, 14.11.2014, A.N. Shukla \& S.K. Srivastava 122767; Near Forest Rest House, 20.11.2014, A.N. Shukla \& S.K. Srivastava 123948.

Spermacoce hispida L., Sp. Pl.: 102. 1753.

On sandy slopes.

Flowering and fruiting: March-November; Kalesar National Park, Compartment - 15, 07.08.2013, A.N. Shukla \& S.K. Srivastava 121909.

Spermacoce pusilla Wall. in Roxb., Fl. Ind. 1: 379. 1820.

Common in sandy and grassy localities.

Flowering and fruiting: August-November; reported here on the basis of Jain \& al. (l.c.).

Spermadictyon suaveolens Roxb., Pl. Coromandel 3(2): 32, t. 236.1815.

On moist places.

Flowering and fruiting: July-December; Kalesar National Park, Compartment - 23, 17.11.2014, A.N. Shukla \& S.K. Srivastava 122799.

\section{ASTERACEAE}

Adenostemma lavenia (L.) Kuntze, Revis. Gen. Pl. 1: 304. 1891. Verbeina lavania L., Sp. Pl.: 902. 1753.

Common near habitation.

Flowering and fruiting: August-November; reported here on the basis of Kumar (l.c.).

Ageratina adenophora (Spreng.) R.M. King \& H. Rob. in Phytologia 19: 211. 1970. Eupatorium adenophorum Spreng., Syst. Veg. 3: 420. 1826.

On dry slopes.

Flowering and fruiting: January-June; Kalesar National Park, along Yamuna River, 09.03.2014, A.N. Shukla \& S.K. Srivastava 122707.

Ageratum conyzoides L., Sp. Pl.: 839. 1753.

On moist places.

Flowering and fruiting: Throughout the year; Kalesar National Park, Compartment - 15, 07.08.2013, A.N. Shukla \& S.K. Srivastava 121905.

Blumea axillaris (Lam.) DC., Prodr. 5: 434. 1836. Conyza axillaris Lam., Encycl. 2: 84. 1786. 
On dry slopes.

Flowering and fruiting: February-April; Kalesar National Park, Compartment - 21, 05.03.2014, A.N. Shukla \& S.K. Srivastava 122124; Kalesar National Park, along Yamuna River, 09.03.2014, A.N. Shukla \& S.K. Srivastava 122198, 122180.

Cyanthillium cinereum (L.) H. Rob., Proc. Biol. Soc. Washington 103(1): 252. 1990. Conyza cinerea L., Sp. Pl.: 862. 1753.

On dry sandy places.

Flowering and fruiting: Throughout the year; Kalesar National Park, Compartment - 1, 06.03.2014, A.N. Shukla \& S.K. Srivastava 122135; Kalesar National Park, Compartment - 2, 07.03.2014, A.N. Shukla \& S.K. Srivastava 122149; Kalesar National Park, Compartment - 17, 12.03.2014, A.N. Shukla \& S.K. Srivastava 122744.

Cyathocline purpurea (Buch.-Ham. ex D. Don) Kuntze, Revis. Gen. Pl. 1: 333. 1891. Tanacetum purpureum Buch.-Ham. ex D. Don, Prodr. Fl. Nepal.: 181. 1825.

On moist places.

Flowering and fruiting: August-March; Kalesar National Park, along Yamuna River, 09.03.2014, A.N. Shukla \& S.K. Srivastava 122704.

Duhaldea cappa (Buch.-Ham. ex D. Don) Pruski \& Anderb. in Compositae Newslett. 40: 44. 2003. Conyza cappa Buch.-Ham. ex D. Don, Prodr. Fl. Nepal.: 176. 1825.

In forest as undergrowth.

Flowering and fruiting: September-November; Kalesar National Park, Compartment - 6, 12.03.2014, A.N. Shukla \& S.K. Srivastava 122737; Kalesar National Park, Compartment - 18, 14.11.2014, A.N. Shukla \& S.K. Srivastava 122763.

Eclipta prostrata (L.) L., Mant. Pl. 2: 286. 1771. Verbesina prostrata L., Sp. Pl.: 902. 1753.

On roadsides in moist places of forest.

Flowering and fruiting: Throughout the year; Kalesar National Park, Kauni line, 12.08.2013, A.N. Shukla \& S.K. Srivastava 122034; Kalesar National Park, Compartment - 17, 04.03.2014, A.N. Shukla \& S.K. Srivastava 122111; Kalesar National Park, Compartment - 24, 17.11.2014, A.N. Shukla \& S.K.
Srivastava 123908.

Emilia sonchifolia (L.) DC. ex DC. in Wight, Contr. Bot. India: 24. 1834. Cacalia sonchifolia L., Sp. Pl.: 835. 1753.

On sandy places.

Flowering and fruiting: January-March; Kalesar National Park, near Kalesar temple, 13.08.2013, A.N. Shukla \& S.K. Srivastava 122058; Compartment - 2, 07.03.2014, A.N. Shukla \& S.K. Srivastava 122147.

Erigeron canadensis L., Sp. Pl.: 863. 1753.

Undergrowth in semi-moist forest.

Flowering and fruiting: June-September; Kalesar National Park, Compartment - 8, 14.11.2014, A.N. Shukla \& S.K. Srivastava 122765.

Erigeron karvinskianus DC., Prodr. 5: 285. 1836.

Rare in the sal forest.

Flowering and fruiting: August-October; reported here on the basis of Jain \& al. (l.c.).

Galinsoga parviflora Cav., Icon 3(2): 41, pl. 281.1795.

On moist places.

Flowering and fruiting: October-March; Kalesar National Park, along Yamuna River, 19.11.2014, A.N. Shukla \& S.K. Srivastava 123933.

Gnaphalium pensylvanicum Willd., Enum. Pl. 2: 867. 1809.

On sandy places.

Flowering and fruiting: January-March; Kalesar National Park, along Yamuna River, 10.03.2014, A.N. Shukla \& S.K. Srivastava 122720.

Helichrysum luteoalbum (L.) Rchb., Handb. Gewächsk., ed. 2, 2: 1460. 1829. Gnaphalium luteoalbum L., Sp. Pl.: 851. 1753.

On sandy places.

Flowering and fruiting: Throughout the year; Kalesar National Park, along Yamuna River, 10.03.2014, A.N. Shukla \& S.K. Srivastava 122719.

Launaea aspleniifolia (Willd.) Hook.f., Fl. Brit. India 3: 415. 1881. Prenanthes aspleniifolia Willd., Sp. Pl. 3: 1540. 1803 .

On sandy slopes. 
Flowering and fruiting: November-March; Kalesar National Park, along Yamuna River, 10.03.2014, A.N. Shukla \& S.K. Srivastava 122731.

Leucomeris spectabilis D. Don, Prodr. Fl. Nepal.: 170. 1825 .

Occasional on rocky slopes.

Flowering and fruiting: April-June; reported here on the basis of Kumar (l.c.).

Myriactis wallichii Less. in Linnaea 6: 129. 1831.

On moist sandy places.

Flowering and fruiting: July-September; Kalesar National Park, along Yamuna River, 10.03.2014, A.N. Shukla \& S.K. Srivastava 122710.

Parthenium hysterophorus L., Sp. Pl.: 988. 1753.

On sandy places.

Flowering and fruiting: September-November; Kalesar National Park, Compartment - 15, 07.08.2013, A.N. Shukla \& S.K. Srivastava 121916.

Pseudoconyza viscosa (Mill.) D'Arcy in Phytologia 25: 281. 1973. Conyza viscosa Mill., Gard. Dict., ed. 8, 8. 1768.

On dry sandy slopes.

Flowering and fruiting: February-May; Kalesar National Park, Compartment - 1, 6.03.2014, A.N. Shukla \& S.K. Srivastava 122132.

Tridax procumbens L., Sp. Pl.: 900. 1753.

On sandy places.

Flowering and fruiting: Throughout the year; Kalesar National Park, Compartment - 15, 07.08.2013, A.N. Shukla \& S.K. Srivastava 121917.

Vicoa indica (L.) DC. in Wight, Contr. Bot. India: 10. 1834. Inula indica L., Sp. Pl., ed. 2: 1236. 1763.

Common in fallow fields and moist places.

Flowering and fruiting: October-March; reported here on the basis of Jain \& al. (l.c.).

Xanthium strumarium L., Sp. Pl.: 987. 1753.

Abundantly found in fallow field, waste places near cultivated fields and on roadsides.

Flowering and fruiting: September-November; reported here on the basis of Jain \& al. (l.c.).

Youngia japonica (L.) DC., Prodr. 7: 194. 1838.
Prenanthes japonica L., Mant. Pl. 107. 1767.

On moist places.

Flowering and fruiting: January-March; Kalesar National Park, Compartment - 6, 12.03.2014, A.N. Shukla \& S.K. Srivastava 122738.

\section{PLUMBAGINACEAE}

Plumbago zeylanica L., Sp. Pl.: 151. 1753.

On forest edges.

Flowering and fruiting: September-April; Kalesar National Park, Compartment - 17, 12.03.2014, A.N. Shukla \& S.K. Srivastava 122741; Near Forest Rest House, 12.03.2014, A.N. Shukla \& S.K. Srivastava 122727.

\section{PRIMULACEAE}

Androsace umbellata (Lour.) Merr. in Philipp. J. Sci. 15: 237. 1919. Drosera umbellata Lour., Fl. Cochinch. 1: 186.1790.

On moist places along nala.

Flowering and fruiting: March-April; Kalesar National Park, Compartment - 17, 04.03.2014, A.N. Shukla \& S.K. Srivastava 122110; Along Yamuna River, 09.03.2014, A.N. Shukla \& S.K. Srivastava 122173.

Lysimachia arvensis (L.) U. Manns \& Anderb. in Willdenowia 39: 51. 2009. Anagallis arvensis L., Sp. Pl.: 148. 1753.

On moist places.

Flowering and fruiting: January-April; Kalesar National Park, Compartment - 17, 04.03.2014, A.N. Shukla \& S.K. Srivastava 122113.

\section{SAPOTACEAE}

Madhuca longifolia J.F. Macbr. var. latifolia (Roxb.) A. Chev. in Rep. Bot. Appl. 23: 149. 1943. Bassia latifolia Roxb., Pl. Coromandel 1: 20, t. 19. 1795.

Common in forest.

Flowering and fruiting: March-June; reported here on the basis of Kumar (l.c.).

\section{EBENACEAE}

Diospyros melanoxylon Roxb., Pl. Coromandel 1: 36, t. 46.1795 . 
On dry forest.

Flowering and fruiting: April-November; Kalesar National Park, Compartment - 18, 15.11.2014, A.N. Shukla \& S.K. Srivastava 122783.

Diospyros montana Roxb., Pl. Coromandel 1(2): 37, t. 48.1795 .

Common in dry habitats.

Flowering and fruiting: March-October; reported here on the basis of Kumar (l.c.).

\section{OLEACEAE}

Jasminum arborescens Roxb., Fl. Ind. 1: 94. 1820.

Common in the hill side forest.

Flowering and fruiting: April-May; reported here on the basis of Jain \& al. (l.c.).

Nyctanthes arbor-tristis L., Sp. Pl.: 6. 1753.

On roadsides.

Flowering and fruiting: September-December; Kalesar National Park, Near Forest Rest House, 09.03.2014, A.N. Shukla \& S.K. Srivastava 122189.

\section{APOCYNACEAE}

Carissa spinarum L., Mant. Pl.: 559. 1771.

On roadsides.

Flowering and fruiting: January-April; Kalesar National Park, near Forest Rest House, 09.03.2014, A.N. Shukla \& S.K. Srivastava 122190.

Cryptolepis buchananii R.Br. ex Roem. \& Schult., Syst. Veg., ed. 15 bis 4: 409. 1819.

Climbing over bushes found along forest roads.

Flowering and fruiting: July-December; reported here on the basis of Jain \& al. (l.c.).

Holarrhena pubescens Wall. ex G. Don, Gen. Hist. 4: 78. 1837.

On roadsides or river bank.

Flowering and fruiting: May-December; Kalesar National Park, Compartment - 17, 11.08.2013, A.N. Shukla \& S.K. Srivastava 122009; Compartment - 22, 16.11.2014, A.N. Shukla \& S.K. Srivastava 122796.

Ichnocarpus frutescens (L.) W.T. Aiton, Hort. Kew., ed. 2, 2: 69. 1811. Apocynum frutescens L., Sp. Pl.: 213. 1753 .
Common, in mixed forests.

Flowering and fruiting: August-December; Kalesar National Park, Compartment - 15, 07.08.2013, A.N. Shukla \& S.K. Srivastava 121903; Compartment - 2, 07.03.2014, A.N. Shukla \& S.K. Srivastava 122156; Near Kalesar village, 19.11.2014, A.N. Shukla \& S.K. Srivastava 123938.

Rauvolfia serpentina (L.) Benth. ex Kurz, Forest Fl. Burma 2: 171. 1877; Hook.f., Fl. Brit. India 3: 632. 1882. Ophioxylon serpentinum L., Sp. Pl.: 1043. 1753.

On moist sandy places.

Flowering and fruiting: July-September; Kalesar National Park, Fire line, Compartment - 10, 16.08.2013, A.N. Shukla \& S.K. Srivastava 122093.

Vallaris solanacea (Roth) Kuntze, Revis. Gen. Pl.: 417. 1891. Peltanthera solanacea Roth, Nov. Pl. Sp.: 132. 1821.

Common on the hilly tracts.

Flowering and fruiting: April-October; reported here on the basis of Jain \& al. (l.c.).

Wrightia arborea (Dennst.) Mabb. in Taxon 26: 533 1977. Periploca arborea Dennst., Schlüssel Hortus Malab.: 15. 1818.

In mixed forests.

Flowering and fruiting: October-May; Kalesar National Park, Compartment - 22, 16.11.2014, A.N. Shukla \& S.K. Srivastava 122794.

\section{BORAGINACEAE}

Cordia dichotoma G. Forst., Fl. Ins. Austr.: 18, n. 110. 1786.

In mixed forest.

Flowering and fruiting: January-July; Kalesar National Park, Compartment - 14, 10.08.2013, A.N. Shukla \& S.K. Srivastava 121977.

Cordia macleodii (Griff.) Hook.f. \& Thomson in J. Proc. Linn. Soc., Bot. 2: 128 1858. Hemigymnia macleodii Griff., Calcutta J. Nat. Hist. 3: 363. 1843.

Rare in hill side forest.

Flowering and fruiting: March-May; reported here on the basis of Jain \& al. (l.c.). 
Cynoglossum lanceolatum Forssk., Fl. Aegypt.-Arab.: 41. 1775.

On sandy slopes.

Flowering and fruiting: September-November; Kalesar National Park, along Sokra River, 10.08.2013, A.N. Shukla \& S.K. Srivastava 121987.

Euploca strigosa (Willd.) Diane \& Hilger in Bot. Jahrb. Syst. 125(1): 49. 2003. Heliotropium strigosum Willd., Sp. Pl. 1: 743. 1798.

On moist places.

Flowering and fruiting: September-May; Kalesar National Park, Compartment - 15, 07.08.2013, A.N. Shukla \& S.K. Srivastava 121901; Along Sokra River, 10.08.2013, A.N. Shukla \& S.K. Srivastava 121982; Along nala, 13.08.2013, A.N. Shukla \& S.K. Srivastava 122045; Along Yamuna River, 09.03.2014, A.N. Shukla \& S.K. Srivastava 122186.

Trichodesma indicum (L.) Lehm., Pl. Asperif. Nucif. 193. 1818. Borago indica L., Sp. Pl.: 137. 1753.

On dry sandy places and rocky slopes.

Flowering and fruiting: September-December; Kalesar National Park, Compartment - 1, 06.03.2014, A.N. Shukla \& S.K. Srivastava 122141; Compartment - 24, 17.11.2014, A.N. Shukla \& S.K. Srivastava 123903.

\section{CONVOLVULACEAE}

Convolvulus prostratus Forssk., Fl. Aegypt.-Arab.: 203. 1775.

Common on roadsides and wasteplaces.

Flowering and fruiting: August-December; reported here on the basis of Negi \& al. (l.c.).

Evolvulus alsinoides (L.) L., Sp. Pl., ed. 2, 1: 392.1762. Convolvulus alsinoides L., Sp. Pl.: 157. 1753.

On dry sandy slopes.

Flowering and fruiting: July-November; Kalesar National Park, Compartment - 15, 07.08.2013, A.N. Shukla \& S.K. Srivastava 121902; Compartment - 1, 06.03.2014, A.N. Shukla \& S.K. Srivastava 122137; Compartment - 8, 14.11.2014, A.N. Shukla \& S.K. Srivastava 122761; Along Yamuna River, 19.11.2014, A.N. Shukla \& S.K. Srivastava 123928.

Evolvulus nummularius (L.) L., Sp. Pl., ed. 2, 1: 391. 1762. Convolvulus nummularius L., Sp. Pl.: 157. 1753.

On moist places.
Flowering and fruiting: March-November; Kalesar National Park, Compartment - 8, 14.11.2014, A.N. Shukla \& S.K. Srivastava 122764.

Ipomoea carnea subsp. fistulosa (Mart. ex Choisy) D.F. Austin, Taxon 26: 237. 1977. Ipomoea fistulosa Mart. ex Choisy, Prodr. 9: 349. 1845.

Along river.

Flowering and fruiting: January-December; Kalesar National Park, Compartment - 8, 14.11.2014, A.N. Shukla \& S.K. Srivastava 122755.

Ipomoea hederifolia L., Syst. Nat., ed. 10, 2: 925.1759.

Common on bushes along forest roads.

Flowering and fruiting: August-December; Kalesar National Park, near Kalesar village, 18.11.2014, A.N. Shukla \& S.K. Srivastava 123918.

Ipomoea nil (L.) Roth, Catal. Bot. 1: 36. 1797. Convolvulus nil L., Sp. Pl., ed. 2: 219. 1762.

Common in outskirts of forests.

Flowering and fruiting: August-December; Kalesar National Park, near forest rest house, 19.11.2014, A.N. Shukla \& S.K. Srivastava 123931.

Porana paniculata Roxb., $\mathrm{Pl}$. Coromandel 3: 31, t. 235. 1819.

Common on bushes.

Flowering and fruiting: September-November; Kalesar National Park, Compartment - 22, 16.11.2014, A.N. Shukla \& S.K. Srivastava 122795.

\section{CUSCUTACEAE}

Cuscuta reflexa Roxb., Pl. Coromandel 2: 3, t. 104. 1798.

Common over small trees and bushes in open areas.

Flowering and fruiting: November-April; Kalesar National Park, near Kalesar village, 18.11.2014, A.N. Shukla \& S.K. Srivastava 123915.

\section{SOLANACEAE}

Datura stramonium L., Sp. Pl.: 179. 1753.

On sandy slopes.

Flowering and fruiting: December-May; Kalesar National Park, along Yamuna River, 10.03.2014, A.N. Shukla \& S.K. Srivastava 122724. 
Nicandra physalodes (L.) Gaertn., Fruct. Sem. Pl. 2: 237. 1791. Atropa physalodes L., Sp. Pl.: 181. 1753.

On roadsides.

Flowering and fruiting: January-March; Kalesar National Park, near Kalesar temple, 14.11.2014, A.N. Shukla \& S.K. Srivastava 123935.

Physalis angulata L., Sp. Pl.: 183. 1753.

In semi-moist places.

Flowering and fruiting: September-December; Kalesar National Park, near Kalesar village, 08.08.2013, A.N. Shukla \& S.K. Srivastava 121941.

Physalis peruviana L., Sp. Pl., ed. 2: 1670. 1763.

Common as undergrowth in forest.

Flowering and fruiting: September-December; Kalesar National Park, along road side, A.N. Shukla 123935.

Solanum americanum Mill., Gard. Dict., ed. 8, 5. 1768.

Near Kalesar temple.

Flowering and fruiting: September-February; Kalesar National Park, Compartment - 24, 18.11.2014, A.N. Shukla \& S.K. Srivastava 123920.

Solanum erianthum D. Don, Prodr. Fl. Nepal.: 96. 1825.

In forest undergrowth.

Flowering and fruiting: Throughout the year; Kalesar National Park, Compartment - 2, 08.03.2014, A.N. Shukla \& S.K. Srivastava 122160.

Solanum incanum L., Sp. Pl.: 188. 1753.

On dry slopes.

Flowering and fruiting: Throughout the year; Kalesar National Park, along Yamuna River, 09.03.2014, A.N. Shukla \& S.K. Srivastava 122192.

Solanum torvum Sw., Prodr.: 47. 1788.

In mixed forest.

Flowering and fruiting: Throughout the year; Kalesar National Park, near Kalesar village, 18.11.2014, A.N. Shukla \& S.K. Srivastava 123914.

Solanum virginianum L., Sp. Pl.: 187. 1753.

On dry sandy places.
Flowering and fruiting: January-June; Along Sokra River, 10.08.2013, A.N. Shukla \& S.K. Srivastava 121978; Kalesar National Park, along Yamuna River, 09.03.2014, A.N. Shukla \& S.K. Srivastava 122199.

\section{SCROPHULARIACEAE}

Bonnaya ciliata (Colsm.) Spreng., Syst. Veg. 1: 41. 1824. Gratiola ciliata Colsm., Prodr. Descr. Gratiol.: 14. 1793.

On moist places.

Flowering and fruiting: August-December; Kalesar National Park, Compartment - 14, 10.08.2013, A.N. Shukla \& S.K. Srivastava 121993B.

Lindenbergia indica (L.) Vatke, Oesterr. Bot. Z. 25: 10. 1875. Dodartia indica L., Sp. Pl.: 633. 1753.

On sandy moist places.

Flowering and fruiting: August-December; Kalesar National Park, along Yamuna River, 10.03.2014, A.N. Shukla \& S.K. Srivastava 122714; Along Yamuna River, 09.03.2014, A.N. Shukla \& S.K. Srivastava 122196.

Lindernia parviflora (Roxb.) Haines, Bot. Bihar Orissa 4: 635. 1922. Gratiola parviflora Roxb., Pl. Coromandel 3: 3. t. 203. 1811.

On moist places.

Flowering and fruiting: September-December; Kalesar National Park, along Yamuna River, 10.03.2014, A.N. Shukla \& S.K. Srivastava 122711.

Mazus pumilus (Burm.f.) Steenis, Nova Guinea n.s., 9: 31. 1958. Lovelia pumila Burm.f., Fl. Ind.: 186, t. 60, f. 3.1768 .

On moist places.

Flowering and fruiting: September-March; Kalesar National Park, along Yamuna River, 09.03.2014, A.N. Shukla \& S.K. Srivastava 122701; Along Yamuna River, 09.03.2014, A.N. Shukla \& S.K. Srivastava 122181; Compartment - 6, 12.03.2014, A.N. Shukla \& S.K. Srivastava 122739.

Mecardonia procumbens (Mill.) Small, Fl. S.E. U.S. 1338. 1903. Erinus procumbens Mill., Gard. Dict., ed. 8, 6. 1768 .

On moist places.

Flowering and fruiting: July-April; Kalesar National Park, along Yamuna River, 10.03.2014, A.N. Shukla \& S.K. Srivastava 122732. 
Scoparia dulcis L., Sp. Pl.: 116. 1753.

In grasslands.

Flowering and fruiting: August-November; Kalesar National Park, compartment-15, 07.08.2013, A.N. Shukla \& S.K. Srivastava 121913.

Torenia cordifolia Roxb., Pl. Coromandel 2(3): 32, t. 161. 1802.

In moist places of the forest.

Flowering and fruiting: August-December; reported here on the basis of Jain \& al. (l.c.).

Torenia crustacea (L.) Cham. \& Schltdl. in Linnaea 2: 570. 1827. Capraria crustacea L., Mant. Pl. 1: 87. 1767.

On moist places.

Flowering and fruiting: August-February; Kalesar National Park, along Yamuna River, 10.08.2013, A.N. Shukla \& S.K. Srivastava 121995; Compartment - 14, 10.08.2013, A.N. Shukla \& S.K. Srivastava 121993A; Along nala in Forest Rest House, 13.08.2013, A.N. Shukla \& S.K. Srivastava 122046; Along Bara River, 13.08.2013, A.N. Shukla \& S.K. Srivastava 122060.

Verbascum coromandelianum (Vahl) Hub.-Mor. in Bauhinia 5: 11. 1973. Celsia coromandeliana Vahl, Symb. Bot. 3: 79. 1794.

On moist places.

Flowering and fruiting: July-March; Kalesar National Park, along Yamuna River, 09.03.2014, A.N. Shukla \& S.K. Srivastava 122179.

Veronica anagallis-aquatica L., Sp. Pl.: 12. 1753.

On moist places.

Flowering and fruiting: October-April; Kalesar National Park, along Yamuna River, 10.03.2014, A.N. Shukla \& S.K. Srivastava 122730.

\section{BIGNONIACEAE}

Fernandoa adenophylla (Wall. ex G. Don) Steenis in Blumea 23: 135. 1976. Bignonia adenophylla Wall. ex G. Don, Gen. Hist. 4: 221. 1838.

On dry places.

Flowering and fruiting: August-November; Kalesar National Park, Compartment - 18, 15.11.2014, A.N. Shukla \& S.K. Srivastava 122771.

Oroxylum indicum (L.) Kurz, Forest Fl. Burma 2: 237.
1877. Bignonia indica L., Sp. Pl.: 625. 1753.

On forest margins.

Flowering and fruiting: September-December; Kalesar National Park, near Kalesar village, 18.11.2014, A.N. Shukla \& S.K. Srivastava 123919.

\section{PEDALIACEAE}

Martynia annua L., Sp. Pl.: 618. 1753.

On roadsides.

Flowering and fruiting: August-October; Kalesar National Park, near Kalesar village, 08.08.2013, A.N. Shukla \& S.K. Srivastava 121940.

\section{ACANTHACEAE}

Barleria cristata L., Sp. Pl.: 636. 1753.

On rocky slopes.

Flowering and fruiting: July-February; Kalesar National Park, Compartment - 24, 17.11.2014, A.N. Shukla \& S.K. Srivastava 123902.

Barleria prionitis L., Sp. Pl.: 636. 1753.

Not common, in waste places.

Flowering and fruiting: September-March; reported here on the basis of Negi \& al. (l.c.).

Blepharis maderaspatensis (L.) B. Heyne ex Roth, Nov. Pl. Sp.: 320. 1821. Acanthus maderaspatensis L., Sp. Pl.: 639. 1753.

On dry slopes.

Flowering and fruiting: November-December; Kalesar National Park, along Yamuna River, 09.03.2014, A.N. Shukla \& S.K. Srivastava 122185.

Dicliptera chinensis (L.) Juss. in Ann. Mus. Natl. Hist. Nat. 9: 268. 1807. Justicia chinensis L., Sp. Pl.: 16. 1753.

Rare, on dry places.

Flowering and fruiting: September-December; Kalesar National Park, Compartment - 21, 05.03.2014, A.N. Shukla \& S.K. Srivastava 122123; Compartment - 2, 07.03.2014, A.N. Shukla \& S.K. Srivastava 122146.

Dicliptera paniculata (Forssk.) I. Darbysh. in Kew Bull. 62: 122. 2007. Dianthera paniculata Forssk., Fl. Aegypt.-Arab.: 7. 1775.

In dry places on roadsides. 
Flowering and fruiting: October-February; Kalesar National Park, Compartment - 1, 06.03.2014, A.N. Shukla \& S.K. Srivastava 122136; near Kalesar village, 18.11.2014, A.N. Shukla \& S.K. Srivastava 123922.

Justicia adhatoda L., Sp. Pl.: 15. 1753.

On roadsides.

Flowering and fruiting: November-July; Kalesar National Park, Compartment - 17, 04.03.2014, A.N. Shukla \& S.K. Srivastava 122104.

Justicia prostrata Gamble, Fl. Madras: 1081. 1924.

On roadsides.

Flowering and fruiting: July-April; Kalesar National Park, Compartment - 17, 11.08.2013, A.N. Shukla \& S.K. Srivastava 122018.

Lepidagathis incurva Buch.-Ham. ex D. Don, Prodr. Fl. Nepal.: 119. 1825.

On dry places.

Flowering and fruiting: November-April; Kalesar National Park, Compartment - 17, 04.03.2014, A.N. Shukla \& S.K. Srivastava 122114; Compartment - 1, 06.03.2014, A.N. Shukla \& S.K. Srivastava 122130.

Rostellularia quinqueangularis (J. Koenig ex Roxb.) Nees in DC., Prodr. 11: 375. 1847. Justicia quinqueangularis J. Koenig ex Roxb., Fl. Ind. 1: 134. 1820 .

On moist places.

Flowering and fruiting: October-December; Kalesar National Park, along Yamuna River, 14.11.2014, A.N. Shukla \& S.K. Srivastava 122936.

Rungia pectinata (L.) Nees in DC., Prodr. 11: 470. 1847. Justicia pectinata L., Cent. Pl.: 3. 1756.

On dry sandy places.

Flowering and fruiting: November-June; Kalesar National Park, Compartment - 1, 06.03.2014, A.N. Shukla \& S.K. Srivastava 122139.

Strobilanthes pavala (Roxb.) J.R.I. Wood in Novon 23: 392. 2014. Ruellia pavala Roxb., Fl. Ind. 3: 47. 1832.

On moist slopes and fire line grasslands.

Flowering and fruiting: October-December; Kalesar National Park, Compartment - 21, 05.03.2014, A.N. Shukla \& S.K. Srivastava 122126; Compartment - 2, 07.03.2014, A.N. Shukla \& S.K. Srivastava 122150.

\section{VERBENACEAE}

Callicarpa macrophylla Vahl, Symb. Bot. 3: 13. 1794.

In moist sandy places and on dry forest edges.

Flowering and fruiting: July-October; Kalesar National Park, Compartment - 15, 07.08.2013, A.N. Shukla \& S.K. Srivastava 121911; Along Sokra River, 10.08.2013, A.N. Shukla \& S.K. Srivastava 121980; Compartment - 17, 20.11.2014, A.N. Shukla \& S.K. Srivastava 123946.

Clerodendrum infortunatum L., Sp. Pl.: 637. 1753.

Undergrowth in forest.

Flowering and fruiting: December-April; Kalesar National Park, Compartment - 3, 08.03.2014, A.N. Shukla \& S.K. Srivastava 122163.

Phyla nodiflora (L.) Greene, Pittonia 4(20): 46.1899. Verbena nodiflora L., Sp. Pl.: 20. 1753.

On eroded steep slopes.

Flowering and fruiting: April-December; Kalesar National Park, along Bara River, 13.08.2013, A.N. Shukla \& S.K. Srivastava 122054.

Premna barbata Wall. ex Schauer in DC., Prodr. 11: 636. 1847.

Not common, in dry scrub miscellaneous forest.

Flowering and fruiting: March-July; reported here on the basis of Negi \& al. (l.c.).

Premna mollissima Roth, Nov. Pl. Sp.: 286. 1821.

On dry slopes.

Flowering and fruiting: April-June; Kalesar National Park, along Yamuna River, 09.03.2014, A.N. Shukla \& S.K. Srivastava 122197; near Kalesar village, 13.03.2014, A.N. Shukla \& S.K. Srivastava 122752.

Pseudocaryopteris bicolor (Roxb. ex Hardw.) P.D. Cantino, Syst. Bot. 23: 381. 1998. Volkameria bicolour Roxb. ex Hardw., Asiat. Res. 6: 366. 1799.

On roadsides.

Flowering and fruiting: January-May; Kalesar National Park, near Kalesar temple, 08.03.2014, A.N. Shukla \& S.K. Srivastava 122161.

Tectona grandis L.f., Suppl. Pl.: 151. 1782.

Planted in the forest.

Flowering and fruiting: September-December; reported 
on the basis of Jain \& al. (l.c.).

Verbena bonariensis L., Sp. Pl.: 29. 1753.

Common along the river bank.

Flowering and fruiting: July-December; reported here on the basis of Jain \& al. (l.c.).

Vitex negundo L., Sp. Pl.: 638. 1753.

On dry places along forest edges and along river bank.

Flowering and fruiting: Throughout the year; Kalesar National Park, Compartment - 15, 07.08.2013, A.N. Shukla \& S.K. Srivastava 121912; Compartment - 23, 17.11.2014, A.N. Shukla \& S.K. Srivastava 123904.

\section{LAMIACEAE}

Anisomeles indica (L.) Kuntze, Revis. Gen. Pl. 2: 512. 1891. Nepeta indica L., Sp. Pl.: 571. 1753.

Common in the forest.

Flowering and fruiting: September-January; reported here on the basis of Jain \& al. (l.c.).

Clinopodium umbrosum (M. Bieb.) K. Koch in Linnaea 21: 673. 1848. Melissa umbrosa M. Bieb., Fl. Taur.-Caucas. 2: 63. 1808.

On sandy moist slopes.

Flowering and fruiting: September-December; Kalesar National Park, along Yamuna River, 10.03.2014, A.N. Shukla \& S.K. Srivastava 122728.

Colebrookea oppositifolia Sm., Exot. Bot. 2: 111, pl. 115. 1806.

On moist slopes.

Flowering and fruiting: November-April; Kalesar National Park, Compartment - 21, 05.03.2014, A.N. Shukla \& S.K. Srivastava 122126.

Mesosphaerum suaveolens (L.) Kuntze, Revis. Gen. Pl. 2: 525. 1891. Ballota suaveolens L., Syst. Nat., ed. 10: 1100. 1759.

On dry places.

Flowering and fruiting: April-November; Kalesar National Park, Compartment - 2, 07.03.2014, A.N. Shukla \& S.K. Srivastava 122152; Compartment - 17, 12.03.2014, A.N. Shukla \& S.K. Srivastava 122743.

Leucas cephalotes (Roth) Spreng., Syst. Veg. 2: 743. 1825. Phlomis cephalotes Roth, Nov. Pl. Sp.: 262. 1821.
On overgrazed places.

Flowering and fruiting: August-November; Kalesar National Park, near Forest Rest House, 13.08.2013, A.N. Shukla \& S.K. Srivastava 122052.

Leucas decemdentata (Willd.) Sm. in Rees, Cycl. 20(2): 6. 1812. Phlomis decemdentata Willd., Sp. Pl. 3: 124. 1800 .

On dry slopes.

Flowering and fruiting: August-March; Kalesar National Park, Compartment - 23, 17.11.2014, A.N. Shukla \& S.K. Srivastava 123905.

Nepeta graciliflora Benth. in Wall., Pl. Asiat. Rar. 1: 65. 1830 .

On moist sandy places.

Flowering and fruiting: August-December; Kalesar National Park, Compartment - 17, 04.03.2014, A.N. Shukla \& S.K. Srivastava 122107; Compartment - 21, 05.03.2014, A.N. Shukla \& S.K. Srivastava 122120; Compartment - 18, 15.11.2014, A.N. Shukla \& S.K. Srivastava 122788.

Pogostemon benghalensis (Burm.f.) Kuntze, Revis. Gen. Pl. 2: 529. 1891. Origanum benghalense Burm.f., Fl. Ind.: 128, t. 38, f. 3. 1768.

Along nala.

Flowering and fruiting: January-April; Kalesar National Park, Compartment - 17, 04.03.2014, A.N. Shukla \& S.K. Srivastava 122105.

\section{PLANTAGINACEAE}

Plantago major L., Sp. Pl.: 112. 1753.

On moist places.

Flowering and fruiting: September-November; Kalesar National Park, along Yamuna River, 10.03.2014, A.N. Shukla \& S.K. Srivastava 122709.

\section{NYCTAGINACEAE}

Boerhavia diffusa L., Sp. Pl.: 3. 1753.

On sandy places.

Flowering and fruiting: August-November; Kalesar National Park, Compartment - 15, 07.08.2013, A.N. Shukla \& S.K. Srivastava 121915; Near Kalesar village, 
18.11.2014, A.N. Shukla \& S.K. Srivastava 123916.

\section{AMARANTHACEAE}

Achyranthes aspera L., Sp. Pl.: 204. 1753.

On roadsides.

Flowering and fruiting: August-January; Kalesar National Park, near Forest Rest House, 09.08.2013, A.N. Shukla \& S.K. Srivastava 121971.

Alternanthera sessilis(L.) R. Br. exDC., Cat.Pl.Horti Monsp. 4: 77. 1813. Gomphrena sessilis L., Sp. Pl.: 225. 1753.

On moist places.

Flowering and fruiting: April-October; Kalesar National Park, near Forest Rest House, 13.08.2013, A.N. Shukla \& S.K. Srivastava 122048.

Amaranthus spinosus L., Sp. Pl.: 991. 1753.

On roadsides in grasslands.

Flowering and fruiting: Throughout the year; Kalesar National Park, Kanni line, 12.08.2013, A.N. Shukla \& S.K. Srivastava 122035; Near Kalesar village, 19.11.2014, A.N. Shukla \& S.K. Srivastava 123934.

Deeringia amaranthoides (Lam.) Merr., Interpr. Herb. Amboin.: 211. 1917. Achyranthes amaranthoides Lam., Encycl. 1: 548. 1785.

On roadsides.

Flowering and fruiting: September-December; Kalesar National Park, near Kalesar temple, 18.11.2014, A.N. Shukla \& S.K. Srivastava 123923.

Gomphrena celosioides Mart., Nova Acta Phys.-Med. Acad. Caes. Leop.-Carol. Nat. Cur. 13(1): 301. 1826.

Common on roadsides.

Flowering and fruiting: July-December; Kalesar National Park, near Forest Rest House, 13.08.2013, A.N. Shukla \& S.K. Srivastava 122041.

Ouret sanguinolenta (L.) Kuntze, Revis. Gen. Pl. 2: 544. 1891. Achyranthes sanguinolenta L., Sp. Pl., ed. 2: 294. 1762 .

On dry sandy places.

Flowering and fruiting: April-October; Kalesar National Park, near Forest Rest House, 08.08.2013, A.N. Shukla \& S.K. Srivastava 121967; Compartment - 1, 06.03.2014, A.N. Shukla \& S.K. Srivastava 122133; Near Kalesar temple, 09.03.2014, A.N. Shukla \& S.K.
Srivastava 122164; Compartment-24, 17.11.2014, A.N. Shukla \& S.K. Srivastava 122800.

Pupalia lappacea (L.) Juss. in Ann. Mus. Natl. Hist. Nat. 2: 132. 1803. Achyranthes lappacea L., Sp. Pl.: 204. 1753.

On moist humus places.

Flowering and fruiting: September-December; Kalesar National Park, Compartment - 15, 07.08.2013, A.N. Shukla \& S.K. Srivastava 121906; near Kalesar village, 18.11.2014, A.N. Shukla \& S.K. Srivastava 123924.

\section{CHENOPODIACEAE}

Chenopodium album L., Sp. Pl.: 219. 1753.

On roadsides.

Flowering and fruiting: January-December; Kalesar National Park, near Kalesar village, 18.11.2014, A.N. Shukla \& S.K. Srivastava 123917.

\section{POLYGONACEAE}

Persicaria barbata (L.) H. Hara, Fl. E. Himalaya: 70. 1966. Polygonum barbatum L., Sp. Pl.: 362. 1753.

On marshy places.

Flowering and fruiting: August-November; Kalesar National Park, along Yamuna River, 19.11.2014, A.N. Shukla \& S.K. Srivastava 123937.

Persicaria glabra (Willd.) M. Gómez, Anales Inst. Segunda Enseñ. 2: 278. 1896. Polygonum glabrum Willd., Sp. Pl. 2: 447. 1799.

Common along the river beds.

Flowering and fruiting: August-March; reported here on the basis of Negi \& al. (l.c.).

Polygonum plebeium R. Br., Prodr.: 420. 1810.

Along water channel.

Flowering and fruiting: November-June; Kalesar National Park, Compartment - 2, 07.03.2014, A.N. Shukla \& S.K. Srivastava 122156.

\section{LAURACEAE}

Litsea glutinosa (Lour.) C.B. Rob. in Philipp. J. Sci. 6(5): 321. 1911. Sebifera glutinosa Lour., Fl. Cochinch. 2: 638.1790 .

Rare in miscellaneous forests. 
Flowering and fruiting: June-October; reported here on the basis of Negi \& al. (l.c.).

\section{LORANTHACEAE}

Dendrophthoe falcata (L.f.) Ettingsh., Denkschr. Kaiserl. Akad. Wiss., Wien. Math.-Naturwiss. Kl. 32: 52. 1872. Loranthus falcatus L.f., Suppl. Pl.: 211. 1782. Parasite on tree.

Flowering and fruiting: September-March; Kalesar National Park, Compartment - 22, 16.11.2014, A.N. Shukla \& S.K. Srivastava 122798.

\section{EUPHORBIACEAE}

Bridelia retusa (L.) A. Juss., Euphorb. Gen.: 109. 1824. Clutia retusa L., Sp. Pl.: 1042. 1753.

Common in dry deciduous forest.

Flowering and fruiting: July-December; reported here on the basis of Kumar (l.c.).

Euphorbia neriifolia L., Sp. Pl.: 451. 1753.

Common on hills.

Flowering and fruiting: October-March; reported here on the basis of Jain \& al. (l.c.).

Euphorbia prolifera Buch.-Ham. ex D. Don, Prodr. Fl. Nepal.: 62. 1825.

Rare in the forest.

Fl \& Fr.: April-July; repoted here on the basis of Jain \& al. (l.c.).

Euphorbia royleana Boiss. in DC., Prodr. 15: 83. 1862. On field border.

Flowering and fruiting: October-December; reported here on the basis of Jain \& al. (l.c.).

Flueggea virosa (Roxb. ex Willd.) Royle, Ill. Bot. Himal. Mts.: 328. 1836. Phyllanthus virosus Roxb. ex Willd., Sp. Pl. 4: 578. 1805.

On hilly slopes.

Flowering and fruiting: September-December; reported here on the basis of Jain \& al. (l.c.).

Jatropha gossypiifolia L., Sp. Pl.: 1006. 1753.

Rare in reserve forest.

Flowering and fruiting: November-May; reported here on the basis of Jain \& al. (l.c.).
Mallotus philippensis (Lam.) Müll.Arg. in Linnaea 34(1): 196. 1865. Croton philippensis Lam., Encycl. 2: 206. 1786.

On roadsides.

Flowering and fruiting: January-October; Kalesar National Park, Compartment - 1, 06.03.2014, A.N. Shukla \& S.K. Srivastava 122129.

Phyllanthus emblica L., Sp. Pl.: 982. 1753.

Occasional in dry deciduous forest.

Flowering and fruiting: March-November; reported here on the basis of Kumar (l.c.).

Phyllanthus urinaria L., Sp. Pl.: 982. 1753.

In sal forest.

Flowering and fruiting: September-December; Kalesar National Park, near Kalesar village, 08.08.2013, A.N. Shukla \& S.K. Srivastava 121948.

Phyllanthus velutinus (Wight) Müll.Arg. in Flora 48: 387. 1865. Glochidion velutinum Wight, Icon. Pl. Ind. Orient.: t. 1907, f. 2.1852.

Common on hillocks.

Flowering and fruiting: March-June; reported here on the basis of Jain \& al. (l.c.).

Ricinus communis L., Sp. Pl.: 1007. 1753.

On roadsides.

Flowering and fruiting: September-June; Kalesar National Park, near Kalesar temple, 10.03.2014, A.N. Shukla \& S.K. Srivastava 122733.

\section{URTICACEAE}

Elatostema sessile J.R. Forst. \& G. Forst., Char. Gen. Pl.: 53. 1775 .

On moist slopes.

Flowering and fruiting: August-October; Kalesar National Park, Compartment - 14, 10.08.2013, A.N. Shukla \& S.K. Srivastava 121990.

\section{ULMACEAE}

Holoptelea integrifolia (Roxb.) Planch. in Ann. Sci. Nat., Bot., Sér. 3, 10: 259. 1848. Ulmus integrifolia Roxb., Pl. Coromandel 1(3): 56. 1796.

Common along forest road sides. 
Flowering and fruiting: January-March; Kalesar National Park, Compartment - 21, 19.11.2014, A.N. Shukla \& S.K. Srivastava 123932.

Trema orientale (L.) Blume, Mus. Bot. 2: 62.1852. Celtis orientalis L., Sp. Pl.: 1044. 1753.

Common on hillocks.

Flowering and fruiting: September-October; reported here on the basis of Jain \& al. (l.c.).

\section{CANNABACEAE}

Cannabis sativa L., Sp. Pl.: 1027. 1753.

On roadsides.

Flowering and fruiting: March-November; Kalesar National Park, near Kalesar village, 08.08.2013, A.N. Shukla \& S.K. Srivastava 121946.

\section{MORACEAE}

Broussonetia papyrifera (L.) L'Hér. ex Vent., Tabl. Regn. Veg. 3: 547. 1799. Morus papyrifera L., Sp. Pl.: 986. 1753.

Planted.

Flowering and fruiting: May-July; reported on the basis of Jain \& al. (l.c.).

Ficus benghalensis L., Sp. Pl.: 1059. 1753.

Along river.

Flowering and fruiting: June-November; Kalesar National Park, Compartment - 21, 16.11.2014, A.N. Shukla \& S.K. Srivastava 122793.

Ficus racemosa L., Sp. Pl.: 1060. 1753.

In mixed forest.

Flowering and fruiting: June-August; Kalesar National Park, Compartment - 17, 19.11.2014, A.N. Shukla \& S.K. Srivastava 123927.

Ficus semicordata Buch.-Ham. ex Sm. in Rees, Cycl. 14: n. 71. 1810.

Not common, in reserve forest.

Flowering and fruiting: July-December; reported on the basis of Jain \& al. (l.c.).

\section{ORCHIDACEAE}

Habenaria plantaginea Lindl., Gen. Sp. Orchid. Pl.: 323. 1835.
On moist places.

Flowering and fruiting: August-September; Kalesar National Park, along seasonal nala situated $1 \mathrm{~km}$ from rest house, $377 \mathrm{~m}, 13.08 .2013$, A.N. Shukla \& S.K. Srivastava 122045.

Zeuxine strateumatica (L.) Schltr. in Bot. Jahrb. Syst. 45: 394. 1911. Orchis strateumatica L., Sp. Pl.: 943. 1753.

On sandy slopes and moist places.

Flowering and fruiting: January-March; Kalesar National Park, Compartment 21, 05.03.2014, A.N. Shukla \& S.K. Srivastava 122117; Along Yamuna River, 09.03.2014, A.N. Shukla \& S.K. Srivastava 122170.

\section{DIOSCOREACEAE}

Dioscorea bulbifera L., Sp. Pl.: 1033. 1753.

In sal forest.

Flowering and fruiting: August-November; Kalesar National Park, Compartment - 13, 12.08.2013, A.N. Shukla \& S.K. Srivastava 122033.

\section{AMARYLLIDACEAE}

Allium cepa L., Sp. Pl.: 300. 1753.

On sandy places.

Flowering and fruiting: January-February; Kalesar National Park, Compartment - 12, 14.08.2013, A.N. Shukla \& S.K. Srivastava 122061.

\section{SMILACACEAE}

Smilax perfoliata Lour., Fl. Cochinch.: 622. 1790.

Common in sal forest on hillocks.

Flowering and fruiting: August-November; reported on the basis of Jain \& al. (l.c.).

Smilax zeylanica L., Sp. Pl.: 1029. 1753.

On moist forest floor.

Flowering and fruiting: June-August; Kalesar National Park, Compartment - 14, 10.08.2013, A.N. Shukla \& S.K. Srivastava 121989.

\section{ASPARAGACEAE}

Asparagus adscendens Roxb., Fl. Ind., ed. 2: 153. 1832. 
In forest undergrowth, climbing on shrubs.

Flowering and fruiting: October-March; Kalesar National Park, near Kalesar temple, 07.03.2014, A.N. Shukla \& S.K. Srivastava 122148; Along Sokra River, 10.08.2013, A.N. Shukla \& S.K. Srivastava 121997.

\section{PONTEDERIACEAE}

Monochoria hastata (L.) Solms in A. DC. \& C. DC., Monogr. Phan. 4: 523. 1883. Pontederia hastata L., Sp. Pl.: 288. 1753.

Common along the margins of River.

Flowering and fruiting: September-December; Kalesar National Park, along Yamuna River, 19.11.2014, A.N. Shukla \& S.K. Srivastava 123925.

\section{COMMELINACEAE}

Commelina caroliniana Walter, Fl. Carol.: 68. 1788.

On moist places.

Flowering and fruiting: October-January; Kalesar National Park, near Kalesar temple, 13.08.2013, A.N. Shukla \& S.K. Srivastava 122059.

Murdannia edulis (Stokes) Faden in Taxon 29(1): 77. 1980. Commelina edulis Stockes, Bot. Mat. Med. 1: 184. 1812.

Rare in sal forest.

Flowering and fruiting: April-May; reported here on the basis of Jain \& al. (l.c.).

Murdannia nudiflora (L.) Brenan in Kew Bull. 7(2): 189. 1952. Commelina nudiflora L., Sp. Pl.: 41.1753.

On moist places.

Flowering and fruiting: August-October; Kalesar National Park, along Sokra River, 10.08.2013, A.N. Shukla \& S.K. Srivastava 121996.

\section{JUNCACEAE}

Juncus bufonius L., Sp. Pl.: 328. 1753.

On moist places.

Flowering and fruiting: January-May; Kalesar National Park, along Yamuna River, 09.03.2014, A.N. Shukla \& S.K. Srivastava 122177.

\section{ARACEAE}

Amorphophallus paeoniifolius (Dennst.) Nicolson in Taxon 26: 338. 1997. Dracontium paeoniifolius Dennst., Schlüssel Hortus Malab.: 13, 21, 38. 1818.

On moist places.

Flowering and fruiting: July-August; Kalesar National Park, along nala in compartment - 17, 13.08.2013, A.N. Shukla \& S.K. Srivastava 122010.

Arisaema tortuosum (Wall.) Schott in Schott \& Endl., Melet. Bot.: 17. 1832. Arum tortuosum Wall., Pl. Asiat. Rar. 2: 10, t. 114. 1830.

On moist forest edges.

Flowering and fruiting: June-November; Kalesar National Park, along forest road side compartment 14, 14.08.2013, A.N. Shukla \& S.K. Srivastava 122067.

Colocasia esculenta (L.) Schott in Schott \& Endl., Melet. Bot.: 18. 1832. Arum esculentum L., Sp. Pl.: 965. 1753.

On moist slopes.

Flowering and fruiting: July-August; Kalesar National Park, along nala in compartment - 17, 11.08.2013, A.N. Shukla \& S.K. Srivastava 122022.

\section{POTAMOGETONACEAE}

Potamogeton nodosus Poir. in Lam., Encycl. Suppl. 4(2): 535.1816.

In ponds and river banks.

Flowering and fruiting: September-March; Kalesar National Park, along Yamuna River, 19.11.2014, A.N. Shukla \& S.K. Srivastava 123926.

\section{CYPERACEAE}

Bolboschoenus maritimus (L.) Palla, Syn. Deut. Schweiz. Fl., ed. 3: 2532. 1905. Scirpus maritimus L., Sp. Pl.: 51. 1753.

On moist places.

Flowering and fruiting: July-October; Kalesar National Park, Compartment - 18, 15.11.2014, A.N. Shukla \& S.K. Srivastava 122774.

Bulbostylis barbata (Rottb.) C.B. Clarke, Fl. Brit. India 6: 651. 1893. Scirpus barbatus Rottb., Descr. Pl. Rar.: 27. 1772 .

On moist places.

Flowering and fruiting: September-November; Kalesar National Park, along Sokra River, 10.08.2013, A.N. 
Shukla \& S.K. Srivastava 121981.

Cyperus brevifolius (Rottb.) Hassk., Cat. Hort. Bot. Bogor. Alt.: 24. 1844. Kyllinga brevifolia Rottb., Descr. Icon. Rar. Pl.: 13, t. 4, f. 3. 1773.

Rarely found along water channels.

Flowering and fruiting: April-June; reported here on the basis of Jain \& al. (l.c.).

Cyperus cyperoides (L.) Kuntze, Revis. Gen. Pl. 3(2): 333. 1898. Scirpus cyperoides L., Mant. Pl.: 181. 1771.

On moist slopes.

Flowering and fruiting: September-December; Kalesar National Park, Compartment - 15, 07.08.2013, A.N. Shukla \& S.K. Srivastava 121914.

Cyperus flavidus Retz., Observ. Bot. 5: 13. 1788.

On marshy places.

Flowering and fruiting: June-September; Kalesar National Park, Compartment - 18, 15.11.2014, A.N. Shukla \& S.K. Srivastava 122775.

Cyperus mindorensis (Steud.) Huygh in Phytotaxa 166(1): 39. 2014. Kyllinga mindorensis Steud., Syn. Pl. Glumac. 2: 67. 1854.

On moist places.

Flowering and fruiting: August-October; Kalesar National Park, Fire line, Compartment - 13, 12.08.2013, A.N. Shukla \& S.K. Srivastava 122026.

Cyperus niveus Retz., Observ. Bot. 5: 12. 1789.

Common on open grassy slopes.

Flowering and fruiting: April-November; reported here on the basis of Negi \& al. (l.c.).

Cyperus pilosus Vahl, Enum. Pl. 2: 354. 1805.

Rare along river bank.

Flowering and fruiting: July-October; reported here on the basis of Jain \& al. (l.c.).

Erioscirpus comosus (Wall.) Palla in Bot. Zeitung (Berlin) 54: 151. 1896. Scirpus comosus Wall., Fl. Ind. 1: 234.1820.

On moist slopes.

Flowering and fruiting: November; Kalesar National Park, along Yamuna River, 09.03.2014, A.N. Shukla \&
S.K. Srivastava 122184; Compartment - 18, 15.11.2014, A.N. Shukla \& S.K. Srivastava 122780.

Fimbristylis schoenoides (Retz.) Vahl, Enum. Pl. 2: 286. 1805. Scirpus schoenoides Retz., Observ. Bot. 5: 14. 1789.

Common in grassy sandy soil.

Flowering and fruiting: July-November; reported here on the basis of Kumar (l.c.).

\section{POACEAE}

Apluda mutica L., Sp. Pl.: 82. 1753.

On dry slopes.

Flowering and fruiting: September-December; Kalesar National Park, Compartment - 13, on fire line, 12.08.2013, A.N. Shukla \& S.K. Srivastava 122032; Compartment - 6, 12.03.2014, A.N. Shukla \& S.K. Srivastava 122736.

Arundo donax L., Sp. Pl.: 81. 1753.

On moist places.

Flowering and fruiting: September-October; Kalesar National Park, Compartment - 8, 14.11.2014, A.N. Shukla \& S.K. Srivastava 122760.

Cenchrus americanus (L.) Morrone in Ann. Bot. (Oxford) 106: 127. 2010. Panicum americanum L., Sp. Pl.: 56. 1753.

On moist places.

Flowering and fruiting: August-January; Kalesar National Park, along Sokra River, 10.08.2013, A.N. Shukla \& S.K. Srivastava 121988.

Chrysopogon zizanioides (L.) Roberty in Bull. Inst. Fondam. Afrique Noire, Sér. A, Sci. Nat. 22: 106. 1960. Phalaris zizanioides L., Mant. Pl.: 183. 1771.

Common in waste places and along forest road.

Flowering and fruiting: August-March; reported on the basis of Jain \& al. (l.c.).

Cynodon dactylon (L.) Pers., Syn. Pl. 1: 85. 1805. Panicum dactylon L., Sp. Pl.: 58. 1753.

On roadsides.

Flowering and fruiting: Throughout the year; Kalesar National Park, near Forest Guest House, 09.08.2013, A.N. Shukla \& S.K. Srivastava 121965.

Dendrocalamus strictus (Roxb.) Nees in Linnaea 9: 
476. 1834. Bambos stricta Roxb., Pl. Coromandel 1(4): 58 , t. 80.1798 .

Near habitation.

Flowering and fruiting: October-December; Kalesar National Park, near Kalesar temple, 13.08.2013, A.N. Shukla \& S.K. Srivastava 122039.

Desmostachya bipinnata (L.) Stapf in Harv., Fl. Cap. 7: 632. 1900. Briza bipinnata L., Syst. Nat., ed. 10, 2: 875. 1759.

Common along the sandy river beds.

Flowering and fruiting: August-November; reported here on the basis of Negi \& al. (l.c.).

Digitaria ciliaris (Retz.) Koeler, Descr. Gram.: 27. 1802. Panicum ciliare Retz., Observ. Bot. 4: 16. 1786.

On roadsides.

Flowering and fruiting: August-October; Near Forest Guest House, 09.08.2013, A.N. Shukla \& S.K. Srivastava 121966; Kalesar National Park, along Sokra River, 10.08.2013, A.N. Shukla \& S.K. Srivastava 121983.

Digitaria longiflora (Retz.) Pers., Syn. Pl. 1: 85. 1805. Paspalum longiflorum Retz., Observ. Bot. 4: 15.1786.

On moist places.

Flowering and fruiting: August-November; Kalesar National Park, along Bala River, 13.08.2013, A.N. Shukla \& S.K. Srivastava 122050.

Eleusine coracana (L.) Gaertn., Fruct. Sem. Pl. 1: 8. 1788. Cynosurus coracanus L., Syst. Nat., ed. 10, 2: 875. 1759.

Common in cultivated field.

Flowering and fruiting: August-October; reported on the basis of Jain \& al. (l.c.).

Eragrostis gangetica (Roxb.) Steud., Syn. Pl. Glumac. 1: 266. 1854. Poa gangetica Roxb., Fl. Ind. 1: 341. 1820.

On roadsides.

Flowering and fruiting: October-November; reported on the basis of Negi \& al. (l.c.).

Eragrostis japonica (Thunb.) Trin., Mem. Acad. Imp. Sci. St.-Petersbourg Ser. 6, Sci. Math. 1: 405. 1831. Poa japonica Thunb., Fl. Jap.: 51. 1784.

On roadsides.

Flowering and fruiting: July-September; reported on the basis of Negi \& al. (l.c.).
Eragrostis unioloides (Retz.) Nees ex Steud., Syn. Pl. Glumac. 1: 264. 1854. Poa unioloides Retz., Observ. Bot. 5: 19. 1789 .

Common in cultivated fields and along forest road.

Flowering and fruiting: November-December; reported here on the basis of Jain \& al. (l.c.).

Eragrostis viscosa (Retz.) Trin. in Mém. Acad. Imp. Sci. St.-Pétersbourg, Sér. 6, Sci. Math. 1: 397. 1830. Poa viscosa Retz., Observ. Bot. 4: 20. 1786.

On sandy places.

Flowering and fruiting: September-November; Kalesar National Park, Compartment - 17, 14.11.2014, A.N. Shukla \& S.K. Srivastava 123941; Compartment - 1, 06.03.2014, A.N. Shukla \& S.K. Srivastava 122143.

Oplismenus compositus (L.) P. Beauv., Ess. Agrostogr.: 54, 168, 169. 1812. Panicum compositum L., Sp. Pl.: 57. 1753.

On moist places.

Flowering and fruiting: September-December; Kalesar National Park, Compartment - 15, 07.08.2013, A.N. Shukla \& S.K. Srivastava 121907; Along Sokra River, 10.08.2013, A.N. Shukla \& S.K. Srivastava 121979.

Phalaris minor Retz., Observ. Bot. 3: 8. 1783.

On moist places.

Flowering and fruiting: January-December; Kalesar National Park, Compartment - 21, 05.03.2014, A.N. Shukla \& S.K. Srivastava 122125.

Phragmites karka (Retz.) Trin. ex Steud., Nomencl. Bot., ed. 2, 2: 324. 1841. Arundo karka Retz., Observ. Bot. 4: 21. 1786 .

On dry slopes.

Flowering and fruiting: October-November; Kalesar National Park, Compartment - 8, 05.03.2014, A.N. Shukla \& S.K. Srivastava 122128; Compartment - 7, 13.03.2014, A.N. Shukla \& S.K. Srivastava 122753; Near Forest Rest House, 20.11.2014, A.N. Shukla \& S.K. Srivastava 123945.

Pogonatherum crinitum (Thunb.) Kunth, Enum. Pl. 1: 478. 1833. Andropogon crinitus Thunb., Fl. Jap.: 40. 1784 .

On steep moist places. 
Flowering and fruiting: July-October; Kalesar National Park, along Bara River, 13.08.2013, A.N. Shukla \& S.K. Srivastava 122049.

Saccharum spontaneum L., Mant. Pl. 2: 183. 1771.

On dry slopes.

Flowering and fruiting: September-November; Kalesar National Park, along nala in Compartment - 17, 11.08.2013, A.N. Shukla \& S.K. Srivastava 122014.

Themeda arundinacea (Roxb.) A. Camus, Fl. IndoChine 7: 363. 1922. Anthistiria arundinacea Roxb., Fl. Ind., ed. 1: 256. 1820.

In forest.

Flowering and fruiting: August-October; reported on the basis of Jain \& al. (l.c.).

Thysanolaena latifolia (Roxb. ex Hornem.) Honda, J. Fac. Sci. Univ. Tokyo, Sect. 3, Bot. 3(1): 312. 1930. Melica latifolia Roxb. ex Hornem., Suppl. Hort. Bot. Hafn.: 17. 1819.

On dry slopes.

Flowering and fruiting: November-May; Kalesar National Park, Fire line, Compartment - 13, 12.08.2013, A.N. Shukla \& S.K. Srivastava 122036; Compartment - 17, 04.03.2014, A.N. Shukla \& S.K. Srivastava 122108.

Urochloa ramosa (L.) T.Q. Nguyen in Novosti Sist. Vyssh. Rast. 3: 13. 1966. Panicum ramosum L., Mant. Pl.: 29. 1767.

On moist places.

Flowering and fruiting: July-November; Kalesar National Park, along Sokra River, 10.08.2013, A.N.
Shukla \& S.K. Srivastava 121984; Along Yamuna River, 09.03.2014, A.N. Shukla \& S.K. Srivastava 122176.

\section{ACKNOWLEDGEMENTS}

The authors are thankful to the Director, Botanical Survey of India, Kolkata and the Head of Office, Botanical Survey of India, Central Regional Centre, Allahabad, for providing facilities and encouragement.

\section{REFERENCES}

DEBTA, M.R. AND S.K. SRIVASTAVA. 2015. Flora of Sultanpur National Park, Gurgaon District, Haryana. Botanical Survey of India, Kolkata, pp. 163.

JAIN, S.P. 1985. Addition to the Flora of North-East Haryana. J. Econ. Taxon. Bot. 6(3): 652-654.

JAIN, S.P. 2004. Floristic Diversity of Haryana - An Assessment of Threatened Plants for Conservation. J. Econ. Taxon. Bot. 28(3): 782-787.

JAIN, S.P., D.M. VERMA, S.C. SINGH, J.S. SINGH AND S. KUMAR. 2000. Flora of Haryana. Central Institute of Medicinal and Aromatic Plants (CIMAP), Lucknow. pp. 1-266.

NEGI, S.S., H.B. NAITHANI, A. CHANDRA AND S.K. KAMBOJ. 2010. Floristic Diversity of shiwalik Hills, Haryana. Forest Research Institute, Dehradun, pp. 352.

KUMAR, S. AND P. NAGIYAN. 2006. Trees and Shrubs of Haryana. Oriental Enterprises, Dehradun. pp. 357.

KUMAR, S. 2001. Flora of Haryana (Materials). Bishen Singh Mahendra Pal Singh, Dehradun. pp. 507. 